\title{
Gravitational self-force by mode sum regularization
}

\author{
Leor Barack \\ Department of Physics, Technion-Israel Institute of Technology, Haifa, 32000, Israel \\ and Albert-Einstein-Institut, Max-Planck-Institut für Gravitationsphysik, Am Mühlenberg 1, D-14476 Golm, Germany
}

(Received 11 May 2001; published 26 September 2001)

\begin{abstract}
We propose a practical scheme for calculating the local gravitational self-force experienced by a test mass particle moving in a black hole spacetime. The method-equally effective for either weak or strong field orbits-employs the mode-sum regularization scheme previously developed for a scalar toy model. The starting point for the calculation, in this approach, is the formal expression for the regularized self-force derived by Mino et al. [Phys. Rev. D 55, 3457 (1997)] (and, independently, by Quinn and Wald [Phys. Rev. D 56, 3381 (1997)]), which involves a worldline integral over the tail part of the retarded Green's function. This force is decomposed into multipole (tensor harmonic) modes, whose sum is subjected to a carefully designed regularization procedure. This procedure involves an analytical derivation of certain "regularization parameters" by means of a local analysis of the Green's function. This paper contains the following main parts: (1) The introduction of the mode sum scheme as applied to the gravitational case. (2) Two simple cases studied: the test case of a static particle in flat spacetime, and the case of a particle at a turning point of a radial geodesic in Schwarzschild spacetime. In both cases we derive all necessary regularization parameters. (3) An analytical foundation is set for applying the scheme in more general cases. (In this paper, the mode sum scheme is formulated within the harmonic gauge. The implementation of the scheme in other gauges shall be discussed in a separate, forthcoming paper.)
\end{abstract}

DOI: $10.1103 /$ PhysRevD.64.084021

PACS number(s): 04.25.- g, 04.30.Db, 04.70.Bw

\section{INTRODUCTION}

A pointlike object of mass $m$ (hereafter a "particle") is known to move along a geodesic of the background spacetime in the limit where $m \rightarrow 0$. If the mass of the particle is finite, the motion would no longer be geodesic: interaction of the particle with its own gravitational field will then give rise to the exertion of a "self-force," pushing the particle away from geodesic motion. (The nonconservative part of this force is customarily referred to as "radiation reaction.") The problem of calculating the gravitational self-force is a long standing one. This problem is usually tackled in the context of perturbation theory, by treating $m$ as a small parameter, and looking for the $O(m)$ correction to the geodesic equation of motion on a fixed background. The prototype problemcalculating the electromagnetic self-force on an accelerating (classical) electron in flat space-was considered by Dirac in his famous 1938 paper [1]. Already in this flat space problem one encounters the fundamental issue of regularization, namely, how to correctly handle the divergence of the electromagnetic field (and self-force) at the particle's location. Dirac's regularization yielded what is now referred to as the "Abraham-Lorentz-Dirac" (ALD) force (proportional to the time derivative of the electron's acceleration). The interpretation of the ALD equation of motion and its solutions has been subject to much further study [2]. A formal framework for a calculation of the electromagnetic self-force in curved spacetime was first developed by DeWitt and Brehme in 1960 [3]. Here, in addition to readdressing the question of regularization, one must also deal with the nonlocal nature of the self-force effect: In curved spacetime, waves emitted by the particle at a given instant may backscatter off the spacetime curvature, and interact back with the particle later on its motion. Consequently, the momentary self-force acting on the particle appears to depend on the particle's entire past history.

The continuous effort for a theoretical characterization of gravitational wave forms from strongly gravitating astrophysical systems led, in recent years, to a rise of interest in the problem of calculating self-forces in curved spacetime. The most prominent consequence of this has been the first development of formal tools for calculating the gravitational self-force (see below). Knowing the self-force is necessary for describing the strong field orbital evolution in various astrophysical scenarios, in particular - the capture of a small compact object by a supermassive black hole [an event expected to serve as a main source of gravitational waves for the future space-based Laser Interferometer Space Antenna (LISA) [4]]. In some occasions, the (time-averaged) orbital evolution, and the consequent gravitational wave forms, may be determined by calculating energy-momentum fluxes at infinity (and through the event horizon) and using balance considerations [5]. This method can be applied successfully in models where the central massive object is spherically symmetric (e.g., a Schwarzschild black hole), or even in the more realistic case of a Kerr background - but then, only for equatorial orbits. In more general cases (a nonequatorial orbit around a Kerr black hole), a full specification of the orbital evolution requires knowledge of the temporal rate of change of the Carter "constant of motion," which, to the best of our knowledge, cannot be achieved by standard balance considerations. In addition, balance considerations involve averaging over orbital periods, and can only account for situations where the orbital evolution is adiabatic. This method is inadequate in other, nonadiabatic scenarios, as the final plunge of the particle into a black hole. In general, therefore, it seems 
that one cannot avoid tackling the problem of calculating the local, momentary self-force.

In 1997, two groups-Mino, Sasaki, and Tanaka (MST) [6] and Quinn and Wald (QW) [7]—have worked out (independently) a formal framework for local gravitational selfforce calculations in curved spacetime. Their combined work presents three different derivations of the self-force, all yielding the same formal expression (though in a different level of rigorousness). In the first approach, MST [6] directly generalized the above mentioned analysis of DeWitt and Brehme to the gravitational case: First the gravitational perturbation induced by the mass particle was evaluated near the worldline using Hadamard's expansion of the retarded Green's function [8]. Then the equation of motion was deduced by imposing local energy-momentum conservation on a thin worldtube surrounding the particle's worldline. The second approach, still by MST [6], employed the technique of matched asymptotic expansion, which is based on considering two asymptotic zones outside the particle: an "internal" zone, where the geometry is taken to be that of a Schwarzschild black hole with a tidal perturbation associated with the background curvature; and an "external" zone, where geometry is that of a perturbation on a fixed background. The equation of motion is then obtained by requiring a consistency of the metric in the matching region of the two zones. The third approach for deriving the gravitational selfforce was presented by QW [7], and is based on what they called the comparison axiom. According to this axiom, the correct self-force can be deduced by appropriately comparing the perturbation on the given curved spacetime with that on flat spacetime. This procedure results in the elimination of the divergent piece of the force, and, presumably, in an extraction of its correct finite part. (QW also applied their approach for calculating the electromagnetic [7] and scalar [9] self-forces.)

As already mentioned, all three derivations of the gravitational self-force yield the same result, which we now briefly describe. In curved spacetime, the two-point retarded Green's function associated with a certain wave equation (i.e., the gravitational perturbation equation in a given gauge) is composed of two parts: an "instantaneous" part, which describes the propagation of influence along the light cone; and the so-called "tail" part, describing the nonlocal effect of waves propagating inside the light cone. The gravitational self-force is derived from the gravitational perturbation produced by the particle, which, it turn, can be expressed as a worldline integral over the Green's function. MST and QW (MSTQW) found that the gravitational self-force (in vacuum, and with no external forces) is due only to the tail part of the Green's function associated with the perturbation equation in the harmonic gauge. That is, the "instantaneous" part of this Green's function yields no contribution to the self-force. [The formal expression for the gravitational selfforce as derived by MSTQW is given in Eq. (4) below.]

Here we may point out two aspects in which the gravitational self-force differs from its electromagnetic (and scalar) counterparts. First, the gravitational self-force contains no local term analogous to the electromagnetic (or scalar) ALD force (which is proportional to the time derivative of the particle's acceleration), as there is no acceleration associated with the purely gravitational field. A more fundamental difference concerns the very nature of the gravitational selfforce as a gauge-dependent entity: When the metric is subjected to a gauge transformation (i.e., an infinitesimal coordinate transformation), additional terms emerge in the particle's equation of motion, which correspond to a change in the effective self-force. (If fact, in the perturbative context the self-force is a "pure gauge" entity, in the sense that it can be locally eliminated by a suitable choice of the gauge.) To provide any meaningful gauge-invariant physical information, the self-force must therefore be supplemented by the metric perturbation to which it corresponds (which, of course, contains all information about the gauge). In this regard, an essential point is that MSTQW's prescription is formulated within the framework of the harmonic gauge. A priori, it is only in this gauge-the harmonic gauge-that the force is guaranteed to be well defined and finite. The analysis presented in the current paper remains within the framework of the harmonic gauge (namely, we shall discuss the "harmonic gauge self-force"). A systematic study of how the self-force behaves under a change of gauge shall be presented in a forthcoming paper [10].

The formal expression obtained by MST and QW for the gravitational self-force was sometimes considered impractical for actual calculations, as it was unclear how one should evaluate the nonlocal tail term in general cases. Also, to apply this expression, one encounters the problem of calculating the metric perturbation (and Green's function) in the harmonic gauge, for which perturbation formalism has not been fully developed as for other customarily used gauges (e.g., the Regge-Wheeler or radiative gauges). The first actual calculation of the gravitational self-force based entirely on the prescription of MST and QW was recently presented by Pfenning and Poisson [11], who considered the motion of a mass particle (as well as scalar and electrically charged particles) moving in a weakly curved region of spacetime. In our present manuscript, the attempt is made to present a practical method for direct implementation of the MST and QW prescription in strong gravitational field.

It should be commented that other approaches to the gravitational self-force, not directly relying on the MST and QW analyses, were also taken recently by a number of authors. Lousto [12] proposed a scheme based on the zetafunction regularization technique, to allow self-force calculation in strong field (Lousto's scheme is similar to the method presented in this paper, in that both methods employ a multipole mode decomposition of the gravitational perturbation). Another method for extracting the finite part of the self-force was proposed by Detweiler [14]. Most recently, Nakano and Sasaki [13] carried out a weak-field calculation of the self-force in a Schwarzschild background by evaluating the tail part of a Green's function. It was assumed, however, that the correct force could be derived from a Green's function associated with a certain Klein-Gordon type wave operator, instead of the harmonic gauge-related Green's function as required in the MST and QW regularization schemes. It is unclear as of yet whether or not the above 
regularization schemes produce, in general, the results that would have been obtained by a strict application of MST and QW regularizations.

As we already mentioned, the calculation scheme to be presented in this manuscript is based on MST and QW's formal expression for the gravitational self-force. It employs a technique-the mode-sum regularization schemepreviously introduced $[15,16]$ and tested $[17-21]$ for the scalar self-force. In general, the application of this scheme consists of two essentially independent parts: in the first part, one expands the perturbation field of the particle into multipole modes (tensor harmonic modes in the gravitational case, for example), and derives the "bare" self-force $l$ modes associated with the $l$ modes of the metric perturbation. The $l$-mode perturbation is finite and continuous even at the particle's location, and the corresponding $l$-mode self-force maintains a finite value as well (although it usually suffers a discontinuity across the worldline). However, the sum over the bare force's $l$ modes turn out to be divergent. In the second part of the mode-sum scheme, certain regularization parameters are calculated analytically, by a local analysis of the Green's function at large $l$ and small spacetime separations. These parameters are then used to regularize the divergent sum over bare force's $l$ modes. This calculation scheme completely relies on MST and QW's results, and contains no further assumptions as to the appropriate regularization (although it does contain certain assumptions concerning the mathematical behavior of various quantities involved in the analysis).

Previously, the above mode-sum scheme was fully implemented in several test cases: A scalar charge held static outside a Schwarzschild black hole [17]; A scalar charge in a circular orbit [18] or one in radial motion $[16,19]$ in Schwarzschild spacetime; and the motion of scalar or electric charges on the background of a massive shell [20]. Recently, Burko and Liu first applied the mode-sum scheme for a static scalar charge in Kerr spacetime [21] (however, an analytical foundation for the scheme has not yet been established in the Kerr case). So far, the mode-sum regularization scheme has not been applied for calculating the gravitational self-force.

The arrangement of this paper is as follows. We start, in Sec. II, by summarizing and discussing MST and QW's result for the regularized gravitational self-force, which is the basis for our calculation scheme. Specializing to the Schwarzschild geometry, in Sec. III we expand the (harmonic gauge) Green's function in tensor harmonics, and obtain a set of equations for its various multipole modes (i.e., a set which does not couple different $l$ and $m$ modes, although it does couple different tensor harmonic components). In Sec. IV we then introduce our mode-sum scheme as applied to the gravitational self-force problem. To demonstrate the applicability of this scheme, in Sec. V we implement it in two simple cases by explicitly calculating all necessary regularization parameters. Section VI contains a summary of our results and a discussion concerning the application of our scheme to other, more general cases.

\section{WORLDLINE INTEGRAL FORMULATION OF THE GRAVITATIONAL SELF-FORCE}

We consider a pointlike particle of mass $m$ moving in the exterior of a black hole with mass $M \gg m .{ }^{1}$ Let the metric $g_{\mu \nu}$ describe the black hole background geometry, which we assume to be a solution of the vacuum Einstein equation (later we specialize our discussion to the Schwarzschild spacetimes). Also let $z^{\alpha}(\tau)$ represent the particle's trajectory, and $u^{\alpha}(\tau) \equiv d z^{\alpha} / d \tau$ stand for its 4-velocity. The particle produces a small perturbation $h_{\alpha \beta} \ll g_{\alpha \beta}$ to the background geometry. The Einstein field equation for the metric $g_{\alpha \beta}$ $+h_{\alpha \beta}$, linearized in $h_{\alpha \beta}$, takes the form

$$
\begin{aligned}
\square \bar{h}_{\alpha \beta}(x)-2 R_{\alpha \beta}^{\mu}{ }^{\nu} \bar{h}_{\mu \nu}(x)= & -16 \pi m \int_{-\infty}^{\infty}(-g)^{-1 / 2} \\
& \times \delta^{4}[x-z(\tau)] u_{\alpha}(\tau) u_{\beta}(\tau) d \tau,
\end{aligned}
$$

where we have introduced the "trace-reversed" metric perturbation

$$
\bar{h}_{\alpha \beta}=h_{\alpha \beta}-\frac{1}{2} g_{\alpha \beta} h
$$

(with $h \equiv g^{\mu \nu} h_{\mu \nu}$ ), and where we have set the harmonic gauge condition $\bar{h}_{: \nu}^{\mu \nu}=0$. In the perturbation equation (1), $\square$ stands for the covariant D'Alembertian operator, $R_{\alpha \beta \gamma \delta}$ is the Riemann tensor in the background geometry, ${ }^{2} g$ stands for the determinant of the metric $g_{\alpha \beta}$, and $\delta$ is the Dirac delta function. The particle does not move along a geodesic of $g_{\alpha \beta}$, as it interacts with its own field $h_{\alpha \beta}$. Phrased differently, to the perturbation $h_{\alpha \beta}$ there corresponds a selfforce $F^{\alpha}$, in terms of which the particle's equation of motion is given by

$$
m a^{\alpha}(\tau)=F^{\alpha}(\tau)
$$

where $a^{\alpha} \equiv u_{; \beta}^{\alpha} u^{\beta}$ is the particle's 4-acceleration, with a semicolon denoting covariant differentiation with respect to the background metric $g_{\alpha \beta}$.

Obviously, the perturbation $h_{\alpha \beta}$ diverges on the worldline itself, and the "bare" self-force associated with this perturbation (as defined below) diverges as well. To obtain the physical equation of motion, one must appropriately regularize the self-force. The analyses by MST and QW [6,7] provide a formal expression for the regularized self-force, to order $O\left(\mathrm{~m}^{2}\right)$, in terms of a worldline integral over derivatives of a retarded Green's function. It is found that, in a vacuum background, $F^{\alpha}$ is solely due to the tail part of the Green's function:

\footnotetext{
${ }^{1}$ The extent to which the concept of a pointlike particle makes sense in the context of the self-force problem is discussed in Ref. [7].

${ }^{2}$ Here we use the convention of Ref. [22] for the Riemann tensor. Note the different convention used by Mino et al. in Ref. [6].
} 


$$
\begin{aligned}
F^{\alpha}(\tau)= & m^{2} k^{\alpha \beta \gamma \delta} \int_{-\infty}^{\tau^{-}} \bar{G}_{\beta \gamma \beta^{\prime} \gamma^{\prime} ; \delta}\left[z^{\mu}(\tau) ; z^{\mu}\left(\tau^{\prime}\right)\right] \\
& \times u^{\beta^{\prime}}\left(\tau^{\prime}\right) u^{\gamma^{\prime}}\left(\tau^{\prime}\right) d \tau^{\prime}
\end{aligned}
$$

Here the "kinematic" tensor $k^{\alpha \beta \gamma \delta}$ is given by

$$
\begin{aligned}
k^{\alpha \beta \gamma \delta}= & \frac{1}{2} g^{\alpha \delta} u^{\beta} u^{\gamma}-g^{\alpha \beta} u^{\gamma} u^{\delta}-\frac{1}{2} u^{\alpha} u^{\beta} u^{\gamma} u^{\delta}+\frac{1}{4} u^{\alpha} g^{\beta \gamma} u^{\delta} \\
& +\frac{1}{4} g^{\alpha \delta} g^{\beta \gamma}
\end{aligned}
$$

(understood to be evaluated at the particle's location), so designed to assure that the self-force has no component tangent to the worldline (i.e., $F^{\alpha} u_{\alpha}=0$ ). This guarantees that the mass $m$ maintains a constant value along the worldline. The quantity $\bar{G}_{\beta \gamma \beta^{\prime} \gamma^{\prime}}$ is the two-point retarded Green's function associated with the wave operator given in the left-hand side of Eq. (1). It satisfies ${ }^{3}$

$$
\begin{aligned}
& \square \bar{G}_{\alpha \beta \alpha^{\prime} \beta^{\prime}}\left(x ; x^{\prime}\right)-2 R_{\alpha \beta}^{\mu}{ }_{\alpha{ }^{\nu}}(x) \bar{G}_{\mu \nu \alpha^{\prime} \beta^{\prime}}\left(x ; x^{\prime}\right) \\
& \quad=-16 \pi(-g)^{-1 / 2} \bar{g}_{\alpha^{\prime}(\alpha}\left(x, x^{\prime}\right) \bar{g}_{\beta) \beta^{\prime}}\left(x, x^{\prime}\right) \delta^{4}\left(x-x^{\prime}\right) \\
& \quad \equiv Z_{\alpha \beta \alpha^{\prime} \beta^{\prime}}
\end{aligned}
$$

with the supplementary causality condition $\bar{G}_{\mu \nu \alpha^{\prime} \beta^{\prime}}\left(x ; x^{\prime}\right)$ $=0$ whenever $x$ lies outside the future light cone of $x^{\prime}$. In this equation, the D'Alembertian operator is taken with respect to $x$, parenthesized indices indicate symmetrization, and $\bar{g}_{\alpha \alpha^{\prime}}$ is the bivector of geodesic parallel displacement defined in Ref. [3]. In what follows we shall need only the value of this bi-vector in the "coincidence" limit: $\lim _{x \rightarrow x^{\prime}} \bar{g}_{\alpha \alpha^{\prime}}=g_{\alpha \alpha^{\prime}}$. Note that the bitensor $\bar{G}_{\mu \nu \alpha^{\prime} \beta^{\prime}}\left(x ; x^{\prime}\right)$ has, in general, 100 independent components (compared with 16 components in the electromagnetic case, and only one in the scalar case). We also mention that the trace-reversed metric perturbation itself is constructed from the Green's function according to

$$
\bar{h}_{\alpha \beta}(x)=m \int_{-\infty}^{\infty} \bar{G}_{\alpha \beta \alpha^{\prime} \beta^{\prime}}\left[x^{\mu} ; z^{\mu}(\tau)\right] u^{\alpha^{\prime}}(\tau) u^{\beta^{\prime}}(\tau) d \tau
$$

To avoid confusion, it is worth commenting here about the different notation previously used by different authors in writing Eq. (4): The trace-reversed Green's function, denoted

\footnotetext{
${ }^{3}$ Note that although the wave operator defining the Green's function $\bar{G}_{\alpha \beta \alpha^{\prime} \beta^{\prime}}$ indeed originates from the perturbation equation in the harmonic gauge, the Green's function itself does not satisfy the harmonic gauge condition, as one can directly verify. (To see this, note that the delta function source does not satisfy the conservation law - a vanishing covariant divergence - as does the source for the metric perturbation itself. Consequently, the harmonic gauge condition is not consistent with the Green's function equation as it is with the perturbation equation.) Note that Eq. (2.12) in Ref. [6] is therefore erroneous.
}

here by $\bar{G}_{\alpha \beta \alpha^{\prime} \beta^{\prime}}$, was denoted $G_{\mu \nu \alpha \beta}^{\mathrm{ret}}$ by $\operatorname{MST}[6], G_{a b a^{\prime} b^{\prime}}^{-}$ by QW [7,23], and $\mathcal{G}_{\alpha \beta \alpha^{\prime} \beta^{\prime}}$ by Pfenning and Poisson [11]. Note that Pfenning and Poisson used a different normalization for the Green's function (it contains an extra factor 4 with respect to either Refs. [6,7] and the normalization used here). Also, note that in Ref. [6] the self-force is expressed in terms of only the tail part of the Green's function, denoted therein by $v_{\alpha \beta \alpha^{\prime} \beta^{\prime}}$, with no contribution from its instantaneous part. This expression, however, is actually identical to the above Eq. (4), as the worldline integral involved is cutoff at $\tau^{-}$.

The Green's function $\bar{G}_{\mu \nu \alpha^{\prime} \beta^{\prime}}$ plays a central role in our analyses. It is important to notice that, based on MST and QW analyses, Eq. (4) is guaranteed to yield the correct, "physical" force, only when using the Green's function defined through Eq. (6). The expression given in Eq. (4) may fail to represent the physical self-force, and may even yield an indefinite result, if a different Green's function is used.

For future use, it is useful to write the Green's function equation (6) in the (noncovariant) form

$$
\square_{s} \bar{G}_{\alpha \beta \alpha^{\prime} \beta^{\prime}}+\mathcal{A}_{\alpha \beta}^{\mu \nu} \bar{G}_{\mu \nu \alpha^{\prime} \beta^{\prime}}=Z_{\alpha \beta \alpha^{\prime} \beta^{\prime}} .
$$

Here $\square_{s}$ stands for the D'Alambertian operator acting on a scalar function,

$$
\square_{s} \equiv \partial_{\alpha} \partial^{\alpha}-g^{\alpha \beta} \Gamma_{\alpha \beta}^{\lambda} \partial_{\lambda}
$$

(where $\Gamma_{\alpha \beta}^{\lambda}$ are the connection coefficients), and the $\mathcal{A}_{\alpha \beta}^{\mu \nu}$, s are certain differential operators, of the first order at most, which describe how the various components of the Green's function couple to each other. In a given coordinate system we have

$$
\begin{aligned}
\mathcal{A}_{\alpha \beta}^{\mu \nu} \bar{G}_{\mu \nu}= & 2 g^{\mu \nu}\left[-2 \Gamma_{(\mu(\alpha}^{\lambda} \bar{G}_{\lambda \beta), \nu)}+\left(R_{\mu(\beta \nu}^{\lambda}\right.\right. \\
& \left.\left.-\Gamma_{\mu \nu,(\beta}^{\lambda}+2 \Gamma_{(\beta(\mu}^{\rho} \Gamma_{\nu) \rho}^{\lambda}\right) \bar{G}_{\alpha) \lambda}+\Gamma_{\nu(\alpha}^{\lambda} \Gamma_{\beta) \mu}^{\rho}\right] \\
& -2 R^{\mu}{ }_{\alpha \beta}{ }^{\nu} \bar{G}_{\mu \nu},
\end{aligned}
$$

where the source-point indices $\alpha^{\prime} \beta^{\prime}$ have been suppressed for brevity. In the Appendix we give explicitly the operators $\mathcal{A}_{\alpha \beta}^{\mu \nu}$ for the Schwarzschild background case (in Schwarzschild coordinates).

\section{TENSOR HARMONIC EXPANSION AND REDUCED EQUATIONS}

Accepting Eq. (4) as the basic expression for the gravitational self-force in curved spacetime, the main concern remains how to implement this expression in actual calculations. One may start by considering limiting cases, as the weak field or slow motion scenarios, in which Eq. (4) could be applied in a direct manner. This, indeed, was done recently by Pfenning and Poisson in Ref. [11]. However, in considering realistic black hole spacetimes, one ultimately wishes to apply Eq. (4) for strong field self-force calculations. Here the introduction of a mode-sum scheme seems inevitable. A mode-sum decomposition approach is neces- 
sary for a dimensional reduction of the problem (in the usual manner), but it is especially beneficial in the context of the self-force problem, because of the following reason: Whereas the metric perturbation diverges on the worldline, its individual modes, as well as the corresponding force modes, maintain a finite value even at the location of the particle. Thus, in exploring the behavior of the individual self-force modes, one avoids dealing with divergent quantities. Still, introducing a mode-sum scheme for calculating the self-force is not a straightforward task: the self-force modes each carries a mixed imprint of both the tail and instantaneous parts of the worldline integral, which results in that the sum over modes usually turns out to diverge. A carefully designed scheme for regularization of the mode sum is thus necessary. The introduction of such a scheme is the main target of this paper.

We first consider the multipole-mode decomposing of the Green's function. As in the rest of this paper, we focus on the spherically symmetric Schwarzschild black hole background, with a line element given by

$$
d s^{2}=-f(r) d t^{2}+f^{-1}(r) d r^{2}+r^{2}\left(d \theta^{2}+\sin ^{2} \theta d \varphi^{2}\right),
$$

where $f(r) \equiv 1-2 M / r$ and $M$ is the black hole's mass. Throughout this paper we use Schwarzschild coordinates $t, r, \theta$, and $\varphi$, relativistic units (with $G=c=1$ ), and metric signature -+++ .

Any (sufficiently regular) symmetric covariant tensor of rank two, $T_{\alpha \beta}$, can be expanded on a 2 -sphere in the form

$$
T_{\alpha \beta}=\sum_{l=0}^{\infty} \sum_{m=-l}^{l} \sum_{i=1}^{10} T^{(i) l m}(r, t) Y_{\alpha \beta}^{(i) l m},
$$

where $T^{(i) l m}$ are scalar coefficients and $Y_{\alpha \beta}^{(i) l m}$ are the ReggeWheeler-Zerilli tensor harmonics [24-27]. In Schwarzschild coordinates $t, r, \theta$, and $\varphi$, the set of tensor harmonics $Y_{\alpha \beta}^{(i) l m}$ is given by ${ }^{4}$

$$
\begin{aligned}
& Y_{\alpha \beta}^{(1) l m}=\left(\begin{array}{cccc}
1 & 0 & 0 & 0 \\
0 & 0 & 0 & 0 \\
0 & 0 & 0 & 0 \\
0 & 0 & 0 & 0
\end{array}\right) Y^{l m}, \\
& Y_{\alpha \beta}^{(2) l m}=i / \sqrt{2}\left(\begin{array}{cccc}
0 & 1 & 0 & 0 \\
1 & 0 & 0 & 0 \\
0 & 0 & 0 & 0 \\
0 & 0 & 0 & 0
\end{array}\right) Y^{l m},
\end{aligned}
$$

${ }^{4}$ Here we adopt the orthogonal set introduced by Zerilli [26], though we use a different notation for the basis tensors: The symbols $Y_{\alpha \beta}^{(1) l m} \ldots Y_{\alpha \beta}^{(10) l m}$ are used here instead of Zerilli's $\mathbf{a}_{l m}^{(0)}, \mathbf{a}_{l m}^{(1)}$, $\mathbf{a}_{l m}, \mathbf{b}_{l m}^{(0)}, \mathbf{b}_{l m}, \mathbf{g}_{l m}, \mathbf{f}_{l m}, \mathbf{c}_{l m}^{(0)}, \mathbf{c}_{l m}$, and $\mathbf{d}_{l m}$, respectively. Note the sign error in Eq. (A2j) of Ref. [26].

$$
\begin{aligned}
& Y_{\alpha \beta}^{(3) l m}=\left(\begin{array}{llll}
0 & 0 & 0 & 0 \\
0 & 1 & 0 & 0 \\
0 & 0 & 0 & 0 \\
0 & 0 & 0 & 0
\end{array}\right) Y^{l m}, \\
& Y_{\alpha \beta}^{(4) l m}=\frac{i r}{\sqrt{2 l(l+1)}}\left(\begin{array}{cccc}
0 & 0 & \partial_{\theta} & \partial_{\varphi} \\
0 & 0 & 0 & 0 \\
\partial_{\theta} & 0 & 0 & 0 \\
\partial_{\varphi} & 0 & 0 & 0
\end{array}\right) Y^{l m}, \\
& Y_{\alpha \beta}^{(5) l m}=\frac{r}{\sqrt{2 l(l+1)}}\left(\begin{array}{cccc}
0 & 0 & 0 & 0 \\
0 & 0 & \partial_{\theta} & \partial_{\varphi} \\
0 & \partial_{\theta} & 0 & 0 \\
0 & \partial_{\varphi} & 0 & 0
\end{array}\right) Y^{l m}, \\
& Y_{\alpha \beta}^{(6) l m}=r^{2} / \sqrt{2}\left(\begin{array}{cccc}
0 & 0 & 0 & 0 \\
0 & 0 & 0 & 0 \\
0 & 0 & 1 & 0 \\
0 & 0 & 0 & s^{2}
\end{array}\right) Y^{l m},
\end{aligned}
$$

$$
Y_{\alpha \beta}^{(7) l m}=\frac{r^{2}}{2 \sqrt{\lambda l(l+1)}}\left(\begin{array}{cccc}
0 & 0 & 0 & 0 \\
0 & 0 & 0 & 0 \\
0 & 0 & D_{2} & D_{1} \\
0 & 0 & D_{1} & -s^{2} D_{2}
\end{array}\right) Y^{l m},
$$

$$
Y_{\alpha \beta}^{(8) l m}=\frac{r}{\sqrt{2 l(l+1)}}\left(\begin{array}{cccc}
0 & 0 & s^{-1} \partial_{\varphi} & -s \partial_{\theta} \\
0 & 0 & 0 & \\
s^{-1} \partial_{\varphi} & 0 & 0 & 0 \\
-s \partial_{\theta} & 0 & 0 & 0
\end{array}\right) Y^{l m},
$$

$$
Y_{\alpha \beta}^{(9) l m}=\frac{i r}{\sqrt{2 l(l+1)}}\left(\begin{array}{cccc}
0 & 0 & 0 & 0 \\
0 & 0 & s^{-1} \partial_{\varphi} & -s \partial_{\theta} \\
0 & s^{-1} \partial_{\varphi} & 0 & 0 \\
0 & -s \partial_{\theta} & 0 & 0
\end{array}\right) Y^{l m},
$$

$$
Y_{\alpha \beta}^{(10) l m}=\frac{-i r^{2}}{2 \sqrt{\lambda l(l+1)}}\left(\begin{array}{cccc}
0 & 0 & 0 & 0 \\
0 & 0 & 0 & 0 \\
0 & 0 & -s^{-1} D_{1} & s D_{2} \\
0 & 0 & s D_{2} & s D_{1}
\end{array}\right) Y^{l m}
$$

where $Y^{l m}(\theta, \varphi)$ are the scalar spherical harmonics, $s$ $\equiv \sin \theta$ and $\lambda \equiv(l-1)(l+2) / 2$, and the operators $D_{1}$ and $D_{2}$ are given by

$$
D_{1} \equiv 2\left(\partial_{\theta}-\cot \theta\right) \partial_{\varphi}, \quad D_{2} \equiv \partial_{\theta \theta}-\cot \theta \partial_{\theta}-s^{-2} \partial_{\varphi \varphi}
$$


The ten ${ }^{5}$ tensor harmonics of Eqs. (13) and (14) constitute an orthogonal set

$$
\begin{gathered}
\int d \Omega \eta^{\alpha \mu} \eta^{\beta \nu}\left[Y_{\mu \nu}^{(i) l m}\right]^{*} Y_{\alpha \beta}^{(j) l^{\prime} m^{\prime}}=k^{(i)} \delta_{i j} \delta_{l l^{\prime}} \delta_{m m^{\prime}} \\
(\text { for } i, j=1, \ldots, 10)
\end{gathered}
$$

where $\eta^{\alpha \mu} \equiv \operatorname{diag}\left[-1,1, r^{-2},(r s)^{-2}\right]$, an asterisk denotes complex conjugation, and the integration is carried over a 2 -sphere (no summation over $i$ is implied on the right-hand side). The coefficient $k^{(i)}$ takes the value -1 for $i=2,4$, and 8 , and +1 otherwise. The seven harmonics $Y_{\alpha \beta}^{(1) l m} \ldots Y_{\alpha \beta}^{(7) l m}$ constitute a basis for all symmetric covariant tensors of even parity, while the remaining three harmonics $Y_{\alpha \beta}^{(8) l m} \ldots Y_{\alpha \beta}^{(10) l m}$ span all odd parity tensors. Recall that the odd and even parity parts of a tensor (e.g., a metric perturbation) are uncoupled, and can be treated separately [24]. Using the orthogonality relation [Eq. (16)], one easily constructs the scalar coefficients of Eq. (12) through

$$
T^{(i) l m}(r, t)=k^{(i)} \int d \Omega \eta^{\alpha \mu} \eta^{\beta \nu}\left[Y_{\mu \nu}^{(i) l m}\right]^{*} T_{\alpha \beta} .
$$

Now, the Green's function $\bar{G}_{\alpha \beta \alpha^{\prime} \beta^{\prime}}\left(x, x^{\prime}\right)$ is a bitensor. It transforms like a tensor at the evaluation point $x$ (when the coordinate transformation is carried out holding $x^{\prime}$ fixed), and it also transforms as a tensor at the source point $x^{\prime}$ (when the transformation is performed with fixed $x$ ). Regarding the Green's function, for a while, as a tensor at the evaluation point $x$, we may expand it in tensor spherical harmonics at that point, as in Eq. (12). We write

$$
\begin{aligned}
\bar{G}_{\alpha \beta \alpha^{\prime} \beta^{\prime}}\left(x, x^{\prime}\right) & =\left(r r^{\prime}\right)^{-1} \sum_{l, m, i} \bar{G}_{\alpha^{\prime} \beta^{\prime}}^{(i) l m}\left(r, t ; x^{\prime}\right) Y_{\alpha \beta}^{(i) l m} \\
& \equiv \sum_{l} \bar{G}_{\alpha \beta \alpha^{\prime} \beta^{\prime}}^{l},
\end{aligned}
$$

where $\bar{G}_{\alpha^{\prime} \beta^{\prime}}^{(i) l m}$ are the multipole expansion coefficients (independent of $\theta$ and $\varphi$ ), the quantity $\bar{G}_{\alpha \beta \alpha^{\prime} \beta^{\prime}}^{l}$ is the one resulting from formally summing over $i$ and $m$, and the radial factor $\left(r r^{\prime}\right)^{-1}$ is introduced for later convenience. We further need to expand the (bitensorial) source term of the Green's function equation (6) in tensor spherical harmonics. This expansion takes the form

$$
Z_{\alpha \beta \alpha^{\prime} \beta^{\prime}}=\left(r r^{\prime}\right)^{-1} \sum_{l, m, i} Z_{\alpha^{\prime} \beta^{\prime}}^{(i) l m}\left(r, t ; x^{\prime}\right) Y_{\alpha \beta}^{(i) l m}
$$

\footnotetext{
${ }^{5}$ Note that for $l=0$ and $l=1$ there are actually fewer independent tensor harmonics: There are only four independent harmonics at $l$ $=0$ (the harmonics $i=1,2,3$, and 6), and eight independent harmonics for each of the three $l=1$ modes (the harmonics $i=7$ and 10 vanish identically at $l=1$ for any value of the azimuthal number $m)$.
}

where, using Eq. (17) with Eq. (6), the coefficients $Z_{\alpha^{\prime} \beta^{\prime}}^{(i) l m}$ are found to be given by

$$
\begin{aligned}
Z_{\alpha^{\prime} \beta^{\prime}}^{(i) l m}= & -16 \pi k^{(i)} \delta\left(t-t^{\prime}\right) \delta\left(r-r^{\prime}\right) \\
& \times g_{\rho^{\prime}\left(\alpha^{\prime}\right.}\left(x^{\prime}\right) g_{\left.\beta^{\prime}\right) \sigma^{\prime}}\left(x^{\prime}\right) \eta^{\rho^{\prime} \mu^{\prime}}\left(x^{\prime}\right) \eta^{\sigma^{\prime} \nu^{\prime}}\left(x^{\prime}\right) \\
& \times\left[Y_{\mu^{\prime} \nu^{\prime}}^{(i) l m}\left(\Omega^{\prime}\right)\right]^{*}
\end{aligned}
$$

(with $\Omega^{\prime}$ standing for $\theta^{\prime}, \varphi^{\prime}$ ). Note here that the coefficients $Z_{\alpha^{\prime} \beta^{\prime}}^{(i) l m}$ depend on the evaluation point $x$ only through the delta functions. ${ }^{6}$

Equation (8) is now separable into multipole modes $l$ and $m$ by means of expansions (18) and (19): By substituting these expansions in Eq. (8) one obtains a set of equations which indeed couple between the ten functions $\bar{G}_{\alpha^{\prime} \beta^{\prime}}^{(i=1 \ldots 10) l m}$ (for given $l, m$ ), but not between the different multipoles $l$ and $m$. To write the equations for the various multipole modes of the Green's function, it is convenient to introduce the Eddington-Finkelstein null coordinates

$$
v \equiv t+r^{*}, \quad u \equiv t-r^{*} \quad\left[\text { where } d r^{*} / d r=f^{-1}(r)\right],
$$

and the time-radial operator

$$
D_{\mathrm{s}}^{l} \equiv \partial_{u v}+V_{\mathrm{s}}^{l}(r)
$$

where

$$
V_{s}^{l}(r)=\frac{f}{4}\left(\frac{f^{\prime}}{r}+\frac{l(l+1)}{r^{2}}\right),
$$

with $f \equiv f(r)$ and $f^{\prime} \equiv d f(r) / d r$. The operator $D_{s}^{l}$ is the familiar wave operator associated with the $l$ mode of a massless scalar field in Schwarzschild spacetime (note the relation $r \square_{s}\left[\phi(r, t) Y^{l m}(\Omega) / r\right]=-4 f^{-1}(r) D_{s}^{l} \phi(r, t) Y^{l m}(\Omega)$, where $\phi(r, t)$ is any function). The equations for the various modes $\bar{G}_{\alpha^{\prime} \beta^{\prime}}^{(i) l m}$ then take the form

$$
D_{\mathrm{s}}^{l} \bar{G}_{\alpha^{\prime} \beta^{\prime}}^{(i) l m}+\mathcal{I}_{(j)}^{(i) l} \bar{G}_{\alpha^{\prime} \beta^{\prime}}^{(j) l m}=S_{\alpha^{\prime} \beta^{\prime}}^{(i) l m} \quad(\text { sum over } j),
$$

where the source term is given by

\footnotetext{
${ }^{6}$ In fact, the naive construction of the coefficients $Z_{\alpha^{\prime} \beta^{\prime}}^{(i) l m}$ yields an expression involving the bi-vector $\bar{g}_{\alpha \beta}$ [as in Eq. (6)]. Form (20) is then obtained by noticing that the coefficients $Z_{\alpha^{\prime} \beta^{\prime}}^{(i) m}$ transform like scalars at the evaluation point $x$. This allows us to prime all tensorial indices of the various factors involved in Eq. (20) (i.e., take these factors to transform like tensors with respect to the source point $x^{\prime}$ ), without affecting the value of $Z_{\alpha^{\prime} \beta^{\prime}}^{(i) l m}$. The presence of the delta functions then further allows us to take all tensorial factors in Eq. (20) to be functions of only the source point coordinates $x^{\prime}$.
} 


$$
\begin{aligned}
S_{\alpha^{\prime} \beta^{\prime}}^{(i) l m}= & -[f(r) / 4] Z_{\alpha^{\prime} \beta^{\prime}}^{(i) l m}=8 k^{(i)} \pi \delta\left(v-v^{\prime}\right) \delta\left(u-u^{\prime}\right) \\
& \times g_{\rho^{\prime}\left(\alpha^{\prime}\right.}\left(x^{\prime}\right) g_{\left.\beta^{\prime}\right) \sigma^{\prime}}\left(x^{\prime}\right) \eta^{\rho^{\prime} \mu^{\prime}}\left(x^{\prime}\right) \eta^{\sigma^{\prime} \nu^{\prime}}\left(x^{\prime}\right) \\
& \times\left[Y_{\mu^{\prime} \nu^{\prime}}^{(i) l m}\left(\Omega^{\prime}\right)\right]^{*},
\end{aligned}
$$

and where $\mathcal{I}_{(j)}^{(i) l}$ are differential operators that couple between different $i$ values. These coupling terms read (suppressing the indices $\alpha^{\prime} \beta^{\prime}$ and $l m$ for brevity)

$$
\begin{array}{rlr}
\mathcal{I}_{(j)}^{(1)} \bar{G}^{(j)}= & \frac{1}{2} f f^{\prime} \bar{G}_{, r}^{(1)}-\frac{1}{8}\left(f^{\prime 2}+4 f f^{\prime} / r\right) \bar{G}^{(1)}+\frac{1}{8}\left(f^{2} f^{\prime 2}\right. & +\frac{1}{4} f^{-1} f^{\prime} i \bar{G}_{, t}^{(8)}-\frac{1}{2}\left(f / r^{2}\right) \sqrt{2 \lambda} \bar{G}^{(10)}, \\
& \left.-2 f^{3} f^{\prime \prime}\right) \bar{G}^{(3)}-\frac{1}{4} \sqrt{2} f f^{\prime}\left(i \bar{G}_{, t}^{(2)}+r^{-1} f \bar{G}^{(6)}\right), & \mathcal{I}_{(j)}^{(10)} \bar{G}^{(j)}=-\frac{1}{2}\left(f / r^{2}\right) \bar{G}^{(10)}-\frac{1}{2}\left(f^{2} / r^{2}\right) \sqrt{2 \lambda} \bar{G}^{(9)} .
\end{array}
$$$$
\mathcal{I}_{(j)}^{(2)} \bar{G}^{(j)}=\frac{1}{4}\left(f^{\prime 2}-f f^{\prime \prime}+2 f^{2} / r^{2}\right) \bar{G}^{(2)}+\frac{1}{4} \sqrt{2} i f f^{\prime}\left(f^{-2} \bar{G}_{, t}^{(1)}\right.
$$$$
\left.+\bar{G}_{, t}^{(3)}\right)-\frac{1}{2} \sqrt{l(l+1)} r^{-2} f \bar{G}^{(4)},
$$$$
\mathcal{I}_{(j)}^{(3)} \bar{G}^{(j)}=-\frac{1}{2} f f^{\prime} \bar{G}_{, r}^{(3)}+\frac{1}{8}\left(8 f^{2} / r^{2}-f^{\prime 2}+4 f f^{\prime} / r\right) \bar{G}^{(3)}
$$$$
+\frac{1}{8}\left(f^{-2} f^{\prime 2}-2 f^{-1} f^{\prime \prime}\right) \bar{G}^{(1)}-\frac{1}{4} \sqrt{2} i f^{-1} f^{\prime} \bar{G}_{, t}^{(2)}
$$$$
-\frac{1}{2} \sqrt{2} r^{-2} f \sqrt{l(l+1)} \bar{G}^{(5)}
$$$$
+\frac{1}{4} \sqrt{2}\left(f^{\prime} / r-2 f / r^{2}\right) \bar{G}^{(6)},
$$$$
\mathcal{I}_{(j)}^{(4)} \bar{G}^{(j)}=\frac{1}{4} f f^{\prime}\left(\bar{G}_{, r}^{(4)}-2 r^{-1} \bar{G}^{(4)}+i \bar{G}_{, t}^{(5)}\right)
$$$$
-\frac{1}{2} \sqrt{l(l+1)}\left(f^{2} / r^{2}\right) \bar{G}^{(2)},
$$$$
\mathcal{I}_{(j)}^{(5)} \bar{G}^{(j)}=-\frac{1}{4} f f^{\prime} \bar{G}_{, r}^{(5)}-\frac{1}{4} f\left(f^{\prime} / r-4 f / r^{2}\right) \bar{G}^{(5)}
$$$$
-\frac{1}{2}\left(f^{2} / r^{2}\right) \sqrt{2 l(l+1)} \bar{G}^{(3)}-\frac{1}{4} f^{-1} f^{\prime} i \bar{G}_{, t}^{(4)}
$$$$
+\frac{1}{2}\left(f / r^{2}\right)\left[\sqrt{l(l+1)} \bar{G}^{(6)}-\sqrt{2 \lambda} \bar{G}^{(7)}\right]
$$$$
\mathcal{I}_{(j)}^{(6)} \bar{G}^{(j)}=\frac{1}{2}\left(f / r^{2}\right)\left(1-2 r f^{\prime}\right) \bar{G}^{(6)}-\frac{\sqrt{2}}{4}\left(f^{\prime} / r\right) \bar{G}^{(1)}
$$$$
-\frac{\sqrt{2}}{4} f^{2}\left(2 f / r^{2}-f^{\prime} / r\right) \bar{G}^{(3)}+\frac{1}{2} \sqrt{l(l+1)}
$$$$
\times\left(f^{2} / r^{2}\right) \bar{G}^{(5)},
$$

$$
\begin{aligned}
& \mathcal{I}_{(j)}^{(7)} \bar{G}^{(j)}=-\frac{1}{2}\left(f / r^{2}\right) \bar{G}^{(7)}-\frac{1}{2}\left(f^{2} / r^{2}\right) \sqrt{2 \lambda} \bar{G}^{(5)}, \\
& \mathcal{I}_{(j)}^{(8)} \bar{G}^{(j)}=\frac{1}{4} f f^{\prime}\left(\bar{G}_{, r}^{(8)}-2 r^{-1} \bar{G}^{(8)}-i \bar{G}_{, t}^{(9)}\right) \\
& \mathcal{I}_{(j)}^{(9)} \bar{G}^{(j)}=-\frac{1}{4} f f^{\prime} \bar{G}_{, r}^{(9)}-\frac{1}{4} f\left(f^{\prime} / r-4 f / r^{2}\right) \bar{G}^{(9)}
\end{aligned}
$$

The separated equations (24) have the convenient property that no coupling between the various modes occur in the main parts of the equations (i.e., the parts containing second derivatives). Coupling between different $i$ modes comes into action only through the $\mathcal{I}_{(j)}^{(i)}$ terms, which contain one or no derivatives. Note that the even parity modes $(i=1 \ldots 7)$ do not couple at all to the odd parity modes $(i=8 \ldots 10)$. The two mode types form a disjoint set of equations, as one would expect.

\section{MODE SUM REGULARIZATION}

Without loss of generality, let us take the point along the particle's trajectory where we wish to calculate the self-force to be at $\tau=0$. Based on Eq. (4), we may express the selfforce as

$$
F_{\alpha}=F_{\alpha}^{(\text {bare })}-F_{\alpha}^{(\text {inst })}
$$

where

$$
\begin{aligned}
F_{\alpha}^{(\text {bare })}= & m^{2} k_{\alpha}^{\beta \gamma \delta} \int_{-\infty}^{0^{+}} \bar{G}_{\beta \gamma \beta^{\prime} \gamma^{\prime} ; \delta}\left[z^{\mu}(\tau=0) ; z^{\mu}\left(\tau^{\prime}\right)\right] \\
& \times u^{\beta^{\prime}}\left(\tau^{\prime}\right) u^{\gamma^{\prime}}\left(\tau^{\prime}\right) d \tau^{\prime} \\
= & m k_{\alpha}{ }^{\beta \gamma \delta} \bar{h}_{\beta \gamma ; \delta}
\end{aligned}
$$

is the "bare" force associated with the metric perturbation $\bar{h}_{\alpha \beta}$ [the second equality here stems from Eq. (7)], and

$$
\begin{aligned}
F_{\alpha}^{(\mathrm{inst})}= & \lim _{\epsilon \rightarrow 0^{+}} \delta F_{\alpha}^{(\epsilon)} \equiv \lim _{\epsilon \rightarrow 0^{+}}\left[m^{2} k_{\alpha}{ }^{\beta \gamma \delta} \int_{-\epsilon}^{0^{+}} \bar{G}_{\beta \gamma \beta^{\prime} \gamma^{\prime} ; \delta}\right. \\
& \left.\times\left[z^{\mu}(\tau=0) ; z^{\mu}\left(\tau^{\prime}\right)\right] u^{\beta^{\prime}}\left(\tau^{\prime}\right) u^{\gamma^{\prime}}\left(\tau^{\prime}\right) d \tau^{\prime}\right]
\end{aligned}
$$

is the "instantaneous" part of the force. The quantities $F_{\alpha}^{\text {(bare) }}$ and $F_{\alpha}^{(\text {inst })}$-both involving integration through the particle's location-are singular and so poorly defined as they stand. For definiteness, we may redefine the integrands appearing in Eqs. (28) and (29) as vector fields in the neighborhood of the evaluation point, and later be interested in their behavior on 
the worldline. Note, however, that the difference $F_{\alpha}^{\text {(bare) }}$ $-F_{\alpha}^{\text {(inst) }}$ does yield a definite finite value at the particle's location: According to the analyses by MST and QW, this value represents the physical self-force $F_{\alpha}$.

To introduce our mode-sum regularization scheme, let us denote by $F_{\alpha}^{l(\text { bare })}$ and $\delta F_{\alpha}^{(\epsilon) l}$, respectively, the contributions to $F_{\alpha}^{(\text {bare })}$ and $\delta F_{\alpha}^{(\epsilon)}$ coming from the $l$ mode of the Green's function [these two quantities are obtained by replacing the Green's function $\bar{G}_{\beta \gamma \beta^{\prime} \gamma^{\prime}}$ in Eqs. (28) and (29) with its $l$-mode $\bar{G}_{\beta \gamma \beta^{\prime} \gamma^{\prime}}^{l}$ defined in Eq. (18)]. We may then express the self-force as a sum over $l$ modes, in the form ${ }^{7}$

$$
F_{\alpha}=\lim _{\epsilon \rightarrow 0^{+}} \sum_{=0}^{\infty}\left(F_{\alpha}^{l(\text { bare })}-\delta F_{\alpha}^{(\epsilon) l}\right) .
$$

An essential feature of our scheme arises from the fact that the $l$ mode of the metric perturbation $h_{\alpha \beta}$ is everywhere finite and continuous; it remains finite and continuous even at the location of the particle, where the overall perturbation diverges. Consequently, the $l$ modes of the bare and instantaneous forces $F_{\alpha}^{l(\text { bare })}$ and $\delta F_{\alpha}^{(\epsilon) l}$ turn out to attain finite values. This behavior has been analyzed and demonstrated in the analogous scalar self-force problem [15,16], and is equally valid in the gravitational case as well (we demonstrate this in Sec. V). As in the scalar self-force model, the two quantities $F_{\alpha}^{l(\text { bare })}$ and $\delta F_{\alpha}^{(\epsilon) l}$ are discontinuous through the particle's location [regarding the integrands appearing in Eqs. (28) and (29) as vector fields in the neighborhood of the particle]. That is, each of these two quantities attains different (finite) values if calculated by taking the limit $r \rightarrow\left(z^{r}\right)^{+}$ or else the limit $r \rightarrow\left(z^{r}\right)^{-}$. [Later we assign to $F_{\alpha}^{l(\text { bare })}$ and $\delta F_{\alpha}^{(\epsilon) l}$ the labels + or - to indicate weather they were calculated from $r \rightarrow\left(z^{r}\right)^{+}$or rather from $r \rightarrow\left(z^{r}\right)^{-}$, respectively.] Note, however, that the difference $F_{\alpha}^{l(\text { bare })}-\delta F_{\alpha}^{(\epsilon) l}$ [as well as the sum over modes in Eq. (30), producing the regularized force $F_{\alpha}$ ] does not depend on the direction from which the limit is taken.

Although each of the bare modes $F_{\alpha}^{l(\text { bare })}$ yields a finite contribution to the self-force, the infinite sum over $F_{\alpha}^{l(\text { bare) }}$ diverges, in general. This is easily demonstrated already in the simple case of a static mass in flat space, a case studied in Sec. V (also see Ref. [16] for a discussion of this point in the analogous scalar case). To carry out the regularization procedure, one seeks an ( $\epsilon$-independent) function $H_{\alpha}^{l \pm}$, such that the series $\Sigma_{l}\left(F_{\alpha}^{l(\text { bare }) \pm}-H_{\alpha}^{l \pm}\right)$ would converge. Once such a function is found, Eq. (30) can be written as

$$
F_{\alpha}=\sum_{l=0}^{\infty}\left(F_{\alpha}^{l(\text { bare }) \pm}-H_{\alpha}^{l \pm}\right)-D_{\alpha}^{ \pm}
$$

where

\footnotetext{
${ }^{7}$ It is assumed here that the differentiation and the integration involved in constructing $F_{\alpha}^{(\text {bare })}$ and $\delta F_{\alpha}^{(\epsilon)}$ out of $\bar{G}_{\beta \gamma \beta^{\prime} \gamma^{\prime}}$ can both be performed term by term with respect to the sum over $l$.
}

$$
D_{\alpha}^{ \pm} \equiv \lim _{\epsilon \rightarrow 0^{+}} \sum_{l=0}^{\infty}\left(\delta F_{\alpha}^{(\epsilon) l \pm}-H_{\alpha}^{l \pm}\right)
$$

The parameter $D_{\alpha}^{ \pm}$is well defined, since, by construction of $H_{\alpha}^{l \pm}$, the difference $\delta F_{\alpha}^{(\epsilon) l \pm}-H_{\alpha}^{l \pm}$ yields a convergent sum over $l$ [note that the two quantities $F_{\alpha}^{l(\text { bare }) \pm}$ and $\delta F_{\alpha}^{(\epsilon) l \pm}$ must bare the same singular behavior at large $l$, as their difference yields a convergent sum over $l$-see Eq. (30)]. Also note here that the limit $\epsilon \rightarrow 0$ and the sum over $l$ should not be interchanged; otherwise, the crucial contribution from $\delta F_{\alpha}^{(\epsilon) l \pm}$ would be lost.

Equation (31) constitutes the basic expression for the gravitational self-force in the framework of the mode sum regularization approach. The implementation of this expression for calculating the self-force at a certain point along a given trajectory involves two (essentially independent) parts: In the first part, one should first calculate (numerically, in general) the $l$ modes of the bare metric perturbation (in the harmonic gauge), and then use these modes to construct the bare force modes $F_{\alpha}^{l \text { (bare) }}$. In the second part of the calculation procedure one should obtain the "regularization function" $H_{\alpha}^{l}$. In principle, this function should be constructed by exploring the asymptotic behavior of the bare modes $F_{\alpha}^{l}$ as $l \rightarrow \infty$. It is more convenient, however, to read this large $l$ asymptotic behavior from the quantity $\delta F_{\alpha}^{(\epsilon) l}$, which is strictly local (recall that $F_{\alpha}^{l(\text { bare) }}$ and $\delta F_{\alpha}^{(\epsilon) l}$ bare the same singular behavior at large $l$ ). To this end, one merely needs the asymptotic behavior of $\delta F_{\alpha}^{(\epsilon) l}$ in the immediate neighborhood of $\epsilon=0$. This allows one to derive $H_{\alpha}^{l}$ (and later also $D_{\alpha}$ ) using local analytical methods, as we shall demonstrate in Sec. V.

In general, the (one-sided values of the) bare modes $F_{\alpha}^{l(\text { (bare })}$ and $\delta F_{\alpha}^{(\epsilon) l}$ are found to diverge at large $l$ as $\propto l$. This was demonstrated for various trajectories in the scalar selfforce problem, and will be demonstrated below in the gravitational case as well. To construct the regularization function $H_{\alpha}^{l}$ so as to regularize the mode sum in Eq. (31), one should therefore derive the three leading-order terms in the $1 / l$ expansion of $\delta F_{\alpha}^{(\epsilon) l}$. It appears more convenient to use an expansion in powers of the new variable

$$
L \equiv l+1 / 2 \text {. }
$$

Denoting the coefficients of the leading-order terms by $A_{\alpha}$, $B_{\alpha}$, and $C_{\alpha}$, we shall have, in general,

$$
H_{\alpha}^{l}=A_{\alpha} L+B_{\alpha}+C_{\alpha} / L .
$$

The implementation of the mode sum regularization scheme therefore amounts to (i) calculating the bare modes $F_{\alpha}^{l \text { (bare) }}$ (this is usually done numerically); (ii) deriving the four "regularization parameters" $A_{\alpha}, B_{\alpha}, C_{\alpha}$, and $D_{\alpha}$ (by local analytical methods); and (iii) summing over $l$ using Eq. (31) to obtain the regularized force $F_{\alpha}$.

The scheme described here is based on the prescription (4), which is formulated within the harmonic gauge. It therefore requires that the bare force modes be obtained from the metric perturbation in the harmonic gauge. This poses a 
problem from a practical point of view, as a separable formalism for the metric perturbation in the harmonic gauge has not been well developed as it has for other gauges [especially the Regge-Wheeler (RW) gauge]. ${ }^{8}$ We shall deal with this gauge problem in a forthcoming paper [10], where an attempt will be made to rephrase the scheme in terms of other gauges, such as the RW gauge.

Before proceeding to discuss some simple applications of the method proposed here, we should comment on a certain technical subtlety which we avoided so far in our discussion. As we thoroughly discuss in Ref. [16] (in the context of the scalar self-force problem), the Green's function does not admit a convergent multipole expansion, as a result of its being singular along the light cone of the source point. As a consequence, the modes $F_{\alpha}^{l(\text { bare) }}$ (and $\delta F_{\alpha}^{(\epsilon) l}$ ) contain certain terms which oscillate rapidly at large $l$, rendering the sums over $l$ in Eqs. (30), (31), and (32) nonconvergent. In Ref. [16] we justified throwing away these divergent oscillatory terms. To formalize the omission of these terms, we therein introduced a new summation and limit operations (the "tilde summation" and "tilde limit") which eliminate any oscillatory divergent terms while preserving the monotonic behavior. The same problem-with the same solution-persists in our current gravitational case. However, to avoid complexity in our current presentation, we shall not attempt to indicate explicitly where a tilde operation is to be applied [as in to above Eqs. (30), (31), and (32)]. In the analysis to follow, a tilde summation or a tilde limit will be implicitly used when appropriate.

\section{SIMPLE APPLICATIONS}

We now demonstrate the applicability of the above calculation scheme in two simple test cases. First we consider the trivial case of a static point mass in flat spacetime. This would provide a simple test case (where the result is obvious: a vanishing self-force) against which we may check the validity of our scheme. We then move on to the Schwarzschild spacetime, and consider a freely falling particle on a radial geodesic. In both cases we construct all four necessary regularization parameters. For simplicity, when considering the second case we shall focus on calculating the force at a presumed turning point of the geodesic (i.e., where $d z^{r} / d \tau$ $=0$ ), for which case the calculation becomes considerably more simple (see below). We emphasize that our calculation and results apply equally well for either weak or strong fields. The application of the scheme in more realistic cases (ones of greater astrophysical relevance) will be presented elsewhere [29].

\section{A. Static particle in flat space}

We consider a static particle of mass $m$ in Minkowski spacetime. The particle is located at $r=r_{0}$, in a certain

\footnotetext{
${ }^{8}$ This problem becomes more acute when dealing with Kerr spacetime, for which a separation formalism for the metric perturbation has been developed so far only in the radiation gauge [28].
}

spherical coordinate system $t, r, \theta, \varphi$. (Here we adopt spherical coordinates in order to make the calculation more closely related to the Schwarzschild case discussed below.) The 4-velocity of this static particle is $u^{\alpha}(\tau)=\delta_{t}^{\alpha}$ at all $\tau$, and thus only the Green's function components $\bar{G}_{\beta \gamma t^{\prime} t^{\prime}}$ take part in constructing the force through Eq. (4). Considering the system of equations (8) for the ten functions $\bar{G}_{\beta \gamma t^{\prime} t^{\prime}}$, one finds that the source $Z_{\alpha \beta t^{\prime} t^{\prime}}$ is nonvanishing only for $\alpha \beta$ $=t t$. [To see this note in Eq. (20) that for $\alpha^{\prime} \beta^{\prime}=t^{\prime} t^{\prime}$ the only contribution comes at $i=1$. Then the only contribution to $Y_{\alpha \beta}^{(1) l m}$ in Eq. (19) is at $\alpha \beta=t t$.] Since all interaction terms $\mathcal{A}_{\alpha \beta}^{t t} \bar{G}_{t t t^{\prime} t^{\prime}}$ appearing in Eqs. (8) vanish in Minkowski spacetime [see Eqs. (A1)-(A10), with $f^{\prime}=0$ ], we find that only the sourced component $G_{t t t^{\prime} t^{\prime}}$ takes a nonzero value, while all other components $\bar{G}_{\beta \gamma t^{\prime} t^{\prime}}$ (which satisfy homogeneous equations) vanish. Thus, for $\alpha^{\prime} \beta^{\prime}=t^{\prime} t^{\prime}$, the system of equations (8) reduces to a single equation for the quantity $\bar{G}_{t t t^{\prime} t^{\prime}} \equiv \bar{G}$ :

$$
\square_{s} \bar{G}=Z_{t t t^{\prime} t^{\prime}}=-16 \pi(-g)^{-1 / 2} \delta^{4}\left(x-x^{\prime}\right) .
$$

Note that in the simple case considered here-a static particle in flat spacetime-only one Green's function component out of 100 actually takes part in the computation of the self-force.

The self-force can now be constructed from Eq. (4) by setting $u^{\alpha}=\delta_{t}^{\alpha}$ and $u^{\alpha^{\prime}}=\delta_{t^{\prime}}^{\alpha^{\prime}}$, and recalling that all component $G_{\beta \gamma t^{\prime} t^{\prime}}$ but $G_{t t t^{\prime} t^{\prime}}$ vanish. We find (evaluating the force at $\tau=0$ without loss of generality)

$$
F_{\alpha}=\frac{1}{4} m^{2} \int_{-\infty}^{0^{-}} \bar{G}_{, \alpha}\left[z^{\mu}(\tau=0) ; z^{\mu}\left(\tau^{\prime}\right)\right] d \tau^{\prime} \quad \text { for } \alpha=r, \theta, \varphi,
$$

as well as $F_{t}=0$. [For $\alpha=t$, the integrand in Eq. (4) in identically zero. This is a trivial result for a static particle, as the force is known to satisfy the normalization condition $F_{\alpha} u^{\alpha}$ $=0$.]

The Green's function equation (35) is exactly the same as the Green's function equation for a scalar field [compare with Eq. (4) in Ref. [16]], apart from a relative numerical factor of 4 on the right-hand side (the source in the gravitational case is greater by 4 than the source in the scalar case). In addition, the construction of the self-force from the Green's function through Eq. (36) is exactly the same as the construction of the tail part of the force in the scalar case [compare with Eqs. (12) and (13) of Ref. [16]], apart from a relative factor of $1 / 4$ on the right-hand side, which compensates for the extra factor of 4 in the Green's function equation. We may conclude, in particular, that the bare modes of the self-force acting on our static particle of mass $m$ are equal to the bare modes of the scalar self-force acting on a static particle of scalar charge $q=m$. It is then possible to simply use the results already obtained in the scalar case: For the regularization parameters we obtain (taking the Minkowski limit of the results described in Refs. $[15,16]$ and replacing $q \rightarrow m$ ) 


$$
A_{\alpha}^{ \pm}=\mp \frac{m^{2}}{r_{0}^{2}} \delta_{\alpha}^{r}, \quad B_{\alpha}=-\frac{m^{2}}{2 r_{0}^{2}} \delta_{\alpha}^{r}, \quad C_{\alpha}=D_{\alpha}=0
$$

As the average of the two sided values of $A_{\alpha}$ vanishes, from Eq. (31) we finally obtain

$$
F_{\alpha}=\sum_{l=0}^{\infty}\left(\bar{F}_{\alpha}^{l(\text { bare })}-B_{\alpha}\right)
$$

where $\bar{F}^{l(\text { bare })} \equiv\left[F^{l(\text { bare })+}+F^{l(\text { bare })-}\right] / 2$.

Now, in our current trivial case, the averaged bare-force modes appearing in Eq. (38) are easily calculated [16]: Solving first for the metric perturbation modes, ${ }^{9}$ one finds that all components of the trace-reversed modes $\bar{h}_{\alpha \beta}^{l}$ vanish, except $\bar{h}_{t t}^{l}$, which is given by $\bar{h}_{t t}^{l}=m r^{-l-1} r_{0}^{l}$ (for $r>r_{0}$ ) and $\bar{h}_{t t}^{l}$ $=m r^{l} r_{0}^{-l-1}$ (for $r<r_{0}$ ). Then, using the second equality of Eq. (28), one obtains $F_{r}^{l+}=-(l+1) m^{2} r_{0}^{-2}$ and $F_{r}^{l-}$ $=l m^{2} r_{0}^{-2}$, yielding $\bar{F}_{r}^{l}=-m^{2} /\left(2 r_{0}^{2}\right)$ (with all other components vanishing). Thus the averaged bare modes of the force are found to be $l$ independent, each identically equal to the regularization parameter $B_{\alpha}$. Consequently, one finds that each of the terms in the sum over $l$ in Eq. (38) vanishes independently, with an obvious (and expected) vanishing of the overall self force.

\section{B. Radial geodesic motion in Schwarzschild, at a turning point}

Let us now consider a particle moving in a radial geodesic in Schwarzschild spacetime. For this orbit we have, identically, $u^{\theta}=u^{\varphi}=0$, and therefore only the Green's function components $\bar{G}_{\alpha \beta t^{\prime} t^{\prime}}, \bar{G}_{\alpha \beta t^{\prime} r^{\prime}}=\bar{G}_{\alpha \beta r^{\prime} t^{\prime}}$, and $\bar{G}_{\alpha \beta r^{\prime} r^{\prime}}$ would take part in constructing the force through Eq. (4). Now, for any given combination of $\alpha^{\prime} \beta^{\prime}$, Eq. (8) constitutes a set of ten coupled equations for the ten independent quantities $\bar{G}_{\alpha \beta \alpha^{\prime} \beta^{\prime}}$. Considering the three sets of equations (8) with $\alpha^{\prime} \beta^{\prime}=t^{\prime} t^{\prime}, t^{\prime} r^{\prime}$, and $r^{\prime} r^{\prime}$, we find that the source $Z_{\alpha \beta \alpha^{\prime} \beta^{\prime}}$ is nonvanishing only for $\alpha \beta=\alpha^{\prime} \beta^{\prime}$ (e.g., in the set of equations for $\bar{G}_{\alpha \beta t^{\prime} t^{\prime}}$, only the equation for $\bar{G}_{t t t^{\prime} t^{\prime}}$ is sourced). Similarly, Eq. (24) forms, for any specific value of $\alpha^{\prime} \beta^{\prime}$, a set of ten equations for the ten tensor-harmonic modes $\bar{G}_{\alpha^{\prime} \beta^{\prime}}^{(i=1 \ldots 10) l m}$. The source $S_{\alpha^{\prime} \beta^{\prime}}^{(i) l m}$ for these equations is nonvanishing only at $i=1$ for $\alpha^{\prime} \beta^{\prime}=t^{\prime} t^{\prime}$, at $i=2$ for $\alpha^{\prime} \beta^{\prime}$ $=t^{\prime} r^{\prime}, r^{\prime} t^{\prime}$, or at $i=3$ for $\alpha^{\prime} \beta^{\prime}=r^{\prime} r^{\prime}$. Clearly, the three odd-parity modes $i=8,9$, and 10, which are not sourced and also do not couple to any of the even parity modes $i=1,2$, and 3, would all vanish (this is expected, of course, by virtue of our physical setup, which only includes an even-parity source). However, the four even-parity modes $i=4,5,6$, and 7 , although not sourced in Eqs. (24), do couple to the modes $i=1,2$, and 3 , and will therefore not vanish, in general.

\footnotetext{
${ }^{9}$ Interestingly, in our case - a static particle in flat spacetime - the metric perturbation in the harmonic gauge exactly coincides with the one in the RW gauge.
}

In conclusion of the above discussion, we find that in the scenario considered here-a particle moving radially on a spherically symmetric background-one has to deal with three sets of seven coupled equations for a total of 21 nontrivial independent components $\bar{G}_{\alpha^{\prime} \beta^{\prime}}^{(i) l m}$ : One of these sets (corresponding to $\alpha^{\prime} \beta^{\prime}=t^{\prime} t^{\prime}$ ) contains a source term only at $i=1$; the other set (corresponding to $\alpha^{\prime} \beta^{\prime}=t^{\prime} r^{\prime}$ or $r^{\prime} t^{\prime}$ ) is sourced only at $i=2$, and the third (for $\alpha^{\prime} \beta^{\prime}=r^{\prime} r^{\prime}$ ) only at $i=3$. To write these three sets of equations in a simple form, it is convenient to redefine our spherical coordinate system, such that the radial trajectory would be directed along the polar axis. In this spherical system, the Green's function (now sourced only at $\theta^{\prime}=0$ ) would contain only axially symmetric, $m=0$ modes. We then also introduce the new variables $\widetilde{G}_{\alpha^{\prime} \beta^{\prime}}^{(i) l}$, defined (for $\alpha^{\prime} \beta^{\prime}=t^{\prime} t^{\prime}, t^{\prime} r^{\prime}, r^{\prime} t^{\prime}$, or $\left.r^{\prime} r^{\prime}\right)$ through

$\bar{G}_{\alpha^{\prime} \beta^{\prime}}^{(i) l} \equiv 8 \pi g_{\rho^{\prime}\left(\alpha^{\prime}\right.}\left(x^{\prime}\right) g_{\left.\beta^{\prime}\right) \sigma^{\prime}}\left(x^{\prime}\right) \eta^{\rho^{\prime} \mu^{\prime}} \eta^{\sigma^{\prime} \nu^{\prime}} a^{(i)} Y^{l}\left(\theta^{\prime}\right) \widetilde{G}_{\mu^{\prime} \nu^{\prime}}^{(i) l}$ (no summation over $i$ ),

where $Y^{l}(\theta) \equiv Y^{l, m=0}$ and $a^{(i)}=1$ for all $i$, except $a^{(2)}$ $=i / \sqrt{2}$. Then each of the above three sets of equations (for $\alpha^{\prime} \beta^{\prime}=t^{\prime} t^{\prime}, t^{\prime} r^{\prime}$, or $r^{\prime} r^{\prime}$ ) takes the form

$$
D_{\mathrm{s}}^{l} \widetilde{G}_{\alpha^{\prime} \beta^{\prime}}^{(i) l}+\widetilde{\mathcal{I}}_{(j)}^{(i) l} \widetilde{G}_{\alpha^{\prime} \beta^{\prime}}^{(j) l}=q_{\alpha^{\prime} \beta^{\prime}}^{(i)} \delta\left(u-u^{\prime}\right) \delta\left(v-v^{\prime}\right),
$$

where

$$
q_{\alpha^{\prime} \beta^{\prime}}^{(i)}= \begin{cases}\delta_{\alpha^{\prime}}^{t^{\prime}} \delta_{\beta^{\prime}}^{t^{\prime}}, & i=1 \\ 2 \delta_{\left(\alpha^{\prime}\right.}^{t^{\prime}} \delta_{\left.\beta^{\prime}\right)}^{r^{\prime}}, & i=2 \\ \delta_{\alpha^{\prime}}^{r^{\prime}} \delta_{\beta^{\prime}}^{r^{\prime}}, & i=3 \\ 0, & i=4, \ldots, 7,\end{cases}
$$

and $\widetilde{\mathcal{I}}_{(j)}^{(i) l}=\mathcal{I}_{(j)}^{(i) l}$ for all $i, j$, except $\widetilde{\mathcal{I}}_{(j=2)}^{(i \neq 2) l}=a^{(2)} \mathcal{I}_{(2)}^{(i) l}$ and $\widetilde{\mathcal{I}}_{(j \neq 2)}^{(i=2) l}=\mathcal{I}_{(j)}^{(2) l} / a^{(2)}$. Finally, to express the $l$-mode Green's function $\bar{G}_{\alpha \beta \alpha^{\prime} \beta^{\prime}}^{l}$ in terms of the new variables $\widetilde{G}_{\alpha^{\prime} \beta^{\prime}}^{(i) l}$, we substitute for $\bar{G}_{\alpha^{\prime} \beta^{\prime}}^{(i) l}$ in Eq. (18), and consider only Green's function components with $\alpha \beta=t t, t r, r r$ (we shall need only these three components in our following analysis). Recalling $Y^{l}(\theta)=[(2 l+1) /(4 \pi)]^{1 / 2} P_{l}(\cos \theta)$ $=[L /(2 \pi)]^{1 / 2} P_{l}(\cos \theta)$ (where $P_{l}$ is the Legendre polynomial) and $P_{l}\left(\cos \theta^{\prime}\right)=1$ for $\theta^{\prime}=0$, for the three relevant components of the $l$-mode Green's function, evaluated at the polar axis $(\theta=0)$, we then obtain

$$
\begin{aligned}
& \bar{G}_{t t \alpha^{\prime} \beta^{\prime}}^{l}=4\left(r r^{\prime}\right)^{-1} f^{2}\left(r^{\prime}\right) L \widetilde{G}_{\alpha^{\prime} \beta^{\prime}}^{(1) l}, \\
& \bar{G}_{t r \alpha^{\prime} \beta^{\prime}}^{l}=-2\left(r r^{\prime}\right)^{-1} L \widetilde{G}_{\alpha^{\prime} \beta^{\prime}}^{(2) l}, \\
& \bar{G}_{r r \alpha^{\prime} \beta^{\prime}}^{l}=4\left(r r^{\prime}\right)^{-1} f^{-2}\left(r^{\prime}\right) L \widetilde{G}_{\alpha^{\prime} \beta^{\prime}}^{(3) l} .
\end{aligned}
$$

[At this stage we may already evaluate the Green's function at the polar axis (where the particle is located), as the following construction of the $r$ component of the force does not 
involve differentiation of the Green's function with respect to either $\theta$ or $\varphi$-see Eq. (43) below.]

To implement our mode sum prescription, we would now like to derive the regularization parameters $A_{\alpha}, B_{\alpha}, C_{\alpha}$, and $D_{\alpha}$. This task turns out to be considerably simpler (though not trivial) when the self-force is evaluated at a presumed turning point of the radial geodesic. As a first nontrivial demonstration of applying our mode-sum regularization scheme, hereafter we focus on this special case. That is, we assume that the geodesic particle is momentarily at rest (i.e., $u^{r}=0$ ) at $r=r_{0}$, and calculate the local self-force at that point. For simplicity, in our calculation we shall consider only the $r$ component of the force. ${ }^{10}$ Extension of this calculation to an arbitrary point of a radial geodesic is straightforward though rather laborious, and will be treated elsewhere [29]. On the technical side, the calculation of the regularization parameters very much resembles the calculation in the scalar case, which we described in much detail in Ref. [16]. For this reason, here we avoid many of the technicalities involved in deriving the parameters, and refer the reader to Ref. [16] for more details. In what follows we only give a very general description of how the analysis proceeds in our current gravitational case.

As explained in Sec. IV, the mode-sum scheme's regularization parameters can be derived by exploring the behavior of the quantity $\delta F_{\alpha}^{(\epsilon) l}$ [see Eq. (29)] at small $\epsilon$, using a perturbation analysis of the Green's function $l$ modes at large- $l$ and small spacetime deviations. In our current problem of a radially moving particle at a turning point, the radial component $\delta F_{r}^{(\epsilon) l}$ is constructed from the various components of the $l$-mode Green's function by

$$
\begin{aligned}
\delta F_{r}^{(\epsilon) l}= & \frac{1}{4} m^{2} f^{-1} \int_{-\epsilon}^{0^{+}}\left[\bar{G}_{t t \alpha^{\prime} \beta^{\prime}, r}^{l}+f^{\prime} f^{-1} \bar{G}_{t t \alpha^{\prime} \beta^{\prime}}^{l}-4 \bar{G}_{r t \alpha^{\prime} \beta^{\prime}, t}^{l}\right. \\
& \left.+f^{2} \bar{G}_{r r \alpha^{\prime} \beta^{\prime}, r}^{l}+3 f f^{\prime} \bar{G}_{r r \alpha^{\prime} \beta^{\prime}}^{l}\right] u^{\alpha^{\prime}}\left(\tau^{\prime}\right) u^{\beta^{\prime}}\left(\tau^{\prime}\right) d \tau^{\prime}
\end{aligned}
$$

(with $f$ and $f^{\prime} \equiv d f / d r$ understood to be evaluated at $r_{0}$ ), which is obtained from Eq. (29) by setting $u^{r}=u^{\theta}=u^{\varphi}=0$ and $u^{t}=f^{-1 / 2}$.

To analyze the large $l$ behavior of the $l$ modes $\bar{G}_{\alpha \beta \alpha^{\prime} \beta^{\prime}}^{l}$ appearing in Eq. (43), we first introduce the "neutralized" spacetime deviation variables ${ }^{11}$

$$
\Delta_{r} \equiv f_{0} L\left(r_{*}-r_{* 0}\right),
$$

\footnotetext{
${ }^{10}$ The angular components $F_{\theta}$ and $F_{\varphi}$ are expected to vanish due to the symmetry of our physical setup, although here we shall not attempt to verify that our scheme indeed leads to this obvious result. At a turning point we also have $F_{t}=0$, stemming, in a trivial manner, from the orthogonality relation $F_{\alpha} u^{\alpha}=0$.

${ }^{11}$ The variables $\Delta_{r}, \Delta_{r^{\prime}}, \Delta_{t}$, and $z$ are "neutral" in the sense that they each consist of a small, $O(\epsilon)$ spacetime deviation, multiplied by the large quantity $L$. The motivation for introducing this kind of variables stems from the detailed discussion in Ref. [16].
}

$$
\begin{aligned}
\Delta_{r^{\prime}} & \equiv f_{0} L\left(r_{*}^{\prime}-r_{* 0}\right), \\
\Delta_{t} & \equiv f_{0} L\left(t-t_{0}\right), \\
\Delta_{t^{\prime}} & \equiv f_{0} L\left(t_{0}-t^{\prime}\right), \\
z & \equiv f_{0} L\left[\left(u-u^{\prime}\right)\left(v-v^{\prime}\right)\right]^{1 / 2},
\end{aligned}
$$

where $(r, t)$ is an off-worldline point in the neighborhood of $r_{0},\left(r^{\prime}, t^{\prime}\right)$ is a worldline point in the past neighborhood of $r_{0}$, and $f_{0} \equiv f^{1 / 2}\left(r_{0}\right) / r_{0}$. We then consider the $l$-mode Green's function as being dependent only on $L$ and the above "neutral" variables, and formally expand the quantities $\widetilde{G}_{\alpha^{\prime} \beta^{\prime}}^{(i) l}$ in powers of $1 / L$, while holding the "neutral" variables fixed:

$$
\widetilde{G}_{\alpha^{\prime} \beta^{\prime}}^{(i) l}=\sum_{k=0}^{\infty} L^{-k} \widetilde{G}_{\alpha^{\prime} \beta^{\prime}[k]}^{(i)}\left(\Delta_{r}, \Delta_{r^{\prime}}, \Delta_{t}, z\right) .
$$

To derive all necessary regularization parameters (including $D_{\alpha}$ ), it is sufficient to obtain an expression for the three leading-order terms in the $1 / L$ expansion of the integrand in Eq. (43). Higher order terms in this expansion do not affect the values of the regularization parameters [16]. By analyzing the Green's function equations (40), we now show that the contribution to $\delta F_{r}^{(\epsilon) l}$ comes at relevant order only from the three components $\bar{G}_{t t t^{\prime} t^{\prime}}^{l}, \bar{G}_{t r t^{\prime} t^{\prime}}^{l}$, and $\bar{G}_{t r t^{\prime} r^{\prime}}^{l}$. In particular, it is shown that the terms of Eq. (43) involving $\bar{G}_{r r \alpha^{\prime} \beta^{\prime}}^{l}$ contribute (for any $\alpha^{\prime} \beta^{\prime}$ ) only at irrelevant high order.

We start by substituting expansion (45) into the set of Green's function equations (40), and pointing out a few useful "rules of thumb:" 12 (i) When the operator $D_{\mathrm{s}}^{l}$ acts on a mode $\widetilde{G}_{\alpha^{\prime} \beta^{\prime}}^{(i) l}$ it "lowers" its order by 2 ; namely, if $\widetilde{G}_{\alpha^{\prime} \beta^{\prime}}^{(i) l}$ $\propto O\left(L^{n}\right)$ (for some $n$ ), then $D_{\mathrm{s}}^{l} \widetilde{G}_{\alpha^{\prime} \beta^{\prime}}^{(i) l} \propto O\left(L^{n+2}\right)$. (ii) A $t$ derivative always acts to lower the order of $\widetilde{G}_{\alpha^{\prime} \beta^{\prime}}^{(i) l}$ by one: if $\widetilde{G}_{\alpha^{\prime} \beta^{\prime}}^{(i) l} \propto O\left(L^{n}\right)$, then $\widetilde{G}_{\alpha^{\prime} \beta^{\prime}, t}^{(i) l} \propto O\left(L^{n+1}\right)$. (iii) An $r$ derivative may lower the order by one at most when acting on a function of both $z$ and $\Delta_{r}$, but does not affect the order when acting on a function of $z$ alone. ${ }^{13}$ (iv) The source terms $\propto \delta\left(u-u^{\prime}\right) \delta\left(v-v^{\prime}\right)$ appearing in Eqs. (40) are of order $L^{2}$. (v) An immediate consequence of all above rules is now

\footnotetext{
${ }^{12}$ In the following discussion we use a terminology according to which the "order" of a mode $\widetilde{G}_{\alpha^{\prime} \beta^{\prime}}^{(i) l}$ is determined by its expansion through Eq. (45), where the "neutral" variables are held fixed. That is, the "order" of $\widetilde{G}_{\alpha^{\prime} \beta^{\prime}}^{(i) l}$ is $L^{-k_{0}}$, where $k_{0}$ is the smallest index $k$ for which $\widetilde{G}_{\alpha^{\prime} \beta^{\prime}[k]}^{(i)}$ is nonvanishing.

${ }^{13}$ This behavior (iii) is special to a turning point. To see this note that at $r=r_{0}$ we have $d z / d r=\left(f_{0} / f\right) L \Delta_{r^{\prime}} / z$. Expanding $\Delta_{r^{\prime}}$ and $z$ in powers of $\tau$ about $\tau\left(r_{0}\right)=0$ and defining the "neutral" proper time $\bar{\tau}$ as in Eq. (50) below, we find, at $\dot{r}=0, \Delta_{r^{\prime}} \cong \frac{1}{2} f_{0}^{-1} \ddot{r} \bar{\tau}^{2} / L$ and $z \cong \bar{\tau}$ (to leading order in $1 / L$ ). Consequently, if $\hat{f}(z)$ is some function of a certain order in $1 / L$, then the $r$ derivative $d \hat{f}(z) / d r$ $=[d \hat{f}(z) / d z](d z / d r)=\frac{1}{2} f^{-1}[d \hat{f}(z) / d z] \ddot{r} \bar{\tau}$ remains of the same order.
} 
apparent from examining Eqs. (40): For any given $i$, a $k$ $=0$ contribution to a mode $\widetilde{G}_{\alpha^{\prime} \beta^{\prime}}^{(i) l}$ can only come from the source term (when nonvanishing), whereas the coupling terms $\widetilde{\mathcal{I}}_{(j)}^{(i) l} \widetilde{G}_{\alpha^{\prime} \beta^{\prime}}^{(j) l}$ contribute only at higher $(k>0)$ order.

First consider Eqs. (40) for $\alpha^{\prime} \beta^{\prime}=t^{\prime} t^{\prime}$. In this case, the only sourced equation is the one for $\widetilde{G}_{t^{\prime} t^{\prime}}^{(1)}$. After substituting expansion (45), we find by the above rule (v) that at the $k$ $=0$ order this equation takes the simple form $D_{\mathrm{s}}^{l} \widetilde{G}_{t^{\prime} t^{\prime}[0]}^{(1) l}$ $=\delta\left(u-u^{\prime}\right) \delta\left(v-v^{\prime}\right)$, with no contribution from the coupling terms $\mathcal{I}_{(j)}^{(1) l} \widetilde{G}_{t^{\prime} t^{\prime}}^{(j) l}$ at this order. The solution to this equation is of order $L^{0}$ [see Eq. (49a) below]; thus $\widetilde{G}_{t^{\prime} t^{\prime}}^{(1) l}$ $\propto O\left(L^{0}\right)$. Now, the mode $\widetilde{G}_{t^{\prime} t^{\prime}}^{(2) l}$ is coupled to $\widetilde{G}_{t^{\prime} t^{\prime}}^{(1) l}$ through the $\bar{G}_{, t}^{(1)}$ term in Eq. (26b). By the above rules (i) and (ii) we thus find $\widetilde{G}_{t^{\prime} t^{\prime}}^{(2)} \propto O\left(L^{-1}\right)$. On the other hand, the mode $\widetilde{G}_{t^{\prime} t^{\prime}}^{(3) l}$ is excited only at $k=2$, through coupling with $\widetilde{G}_{t^{\prime} t^{\prime}}^{(1) l}$ and $\widetilde{G}_{t^{\prime} t^{\prime}, t}^{(2) l}$-see Eq. (26c). We find further that $\widetilde{G}_{t^{\prime} t^{\prime}}^{(4,5) l}$ $\propto O\left(L^{-3}\right), \widetilde{G}_{t^{\prime} t^{\prime}}^{(6)} \propto O\left(L^{-2}\right)$, and $\widetilde{G}_{t^{\prime} t^{\prime}}^{(7)} \propto O\left(L^{-4}\right)$. It can be easily checked now that, up to $O\left(L^{-2}\right)$, the first two equations of the set of equations (24) - the ones with $i=1$ and 2 -form a closed set of equations, with coupling to other modes affecting only at higher orders:

$$
\begin{gathered}
D_{s} \widetilde{G}_{t^{\prime} t^{\prime}}^{(1) l}+\frac{1}{2} f f^{\prime} \widetilde{G}_{t^{\prime} t^{\prime}, r}^{(1) l}-\frac{1}{8}\left(f^{\prime 2}+4 f f^{\prime} / r\right) \widetilde{G}_{t^{\prime} t^{\prime}}^{(1) l}+\frac{1}{4} f f^{\prime} \widetilde{G}_{t^{\prime} t^{\prime}, t}^{(2) l} \\
=\delta\left(v-v^{\prime}\right) \delta\left(u-u^{\prime}\right), \\
D_{s} \widetilde{G}_{t^{\prime} t^{\prime}}^{(2) l}+\frac{1}{2} f^{-1} f^{\prime} \widetilde{G}_{t^{\prime} t^{\prime}, t}^{(1) l}=0 .
\end{gathered}
$$

Using Eq. (42) we may now evaluate the order of $l$ modes $\bar{G}_{\alpha \beta t^{\prime} t^{\prime}}^{l}$, and proceed to evaluate the order of the five integrand terms involved in constructing $\delta F_{r}^{(\epsilon) l}$ through Eq. (43). Using rules (ii) and (iii) and recalling $u^{t^{\prime}} \propto O\left(L^{0}\right)$, we find that the first integrand term in Eq. (43) is of order $L^{2}$, the second and third terms are of order $L^{1}$, and the last two terms (the ones involving $\bar{G}_{r r t^{\prime} t^{\prime}}^{l}$ ) are only of order $L^{-1}$. Since only integrand terms up to $O\left(L^{0}\right)$ are necessary for calculating the regularization parameters, we conclude that the component $\bar{G}_{r r t^{\prime} t^{\prime}}^{l}$ would be of no relevance for this calculation. Up to the necessary order, the set of Green's function equations (40) therefore reduces, in the case $\alpha^{\prime} \beta^{\prime}=t^{\prime} t^{\prime}$, to a closed-form set for $\widetilde{G}_{t^{\prime} t^{\prime}}^{(1) l}$ and $\widetilde{G}_{t^{\prime} t^{\prime}}^{(2) l}$, given by Eq. (46).

Next consider the set of equations (40) for $\alpha^{\prime} \beta^{\prime}=t^{\prime} r^{\prime}$. Here only the $i=2$ component is sourced, and we find $\widetilde{G}_{t^{\prime} r^{\prime}}^{(2) l} \propto O\left(L^{0}\right)$. The modes $i=1$ and 3 are sourced by $\widetilde{G}_{t^{\prime} r^{\prime}, t}^{(2) l}$, leading to $\widetilde{G}_{t^{\prime} r^{\prime}}^{(1,3) l} \propto O\left(L^{-1}\right)$. One similarly finds that the mode $i=4$ is of order $L^{-1}$, the mode $i=5$ is of order $L^{-2}$, and the modes $i=6$ and 7 are only of order $L^{-3}$. Again, we use Eq. (42) to evaluate the order of each of the five integrand terms appearing in Eq. (43), this time for $\alpha^{\prime} \beta^{\prime}$ $=t^{\prime} r^{\prime}$. We now recall, however, that $u^{r^{\prime}}$ vanishes at $r_{0}$, and is therefore of order $u^{r^{\prime}} \propto \ddot{r} / L$. It is then easily shown that the only relevant integrand term is $\bar{G}_{t r r^{\prime} t^{\prime}, t}^{l} u^{t^{\prime}} u^{r^{\prime}} \propto O\left(L^{1}\right)$, while each of the four other terms contribute to the integral in Eq.
(43) only at order $L^{-2}$, which is irrelevant for calculating the regularization parameters. Moreover, one finds that at the relevant order, the mode $\widetilde{G}_{t^{\prime} r^{\prime}}^{(2) l}$ satisfies a single closed-form equation

$$
D_{s} \widetilde{G}_{t^{\prime} r^{\prime}}^{(2) l}=\delta\left(v-v^{\prime}\right) \delta\left(u-u^{\prime}\right) .
$$

(This equation happens to coincide with the scalar Green's function equation.)

Finally, let us consider Eqs. (40) for $\alpha^{\prime} \beta^{\prime}=r^{\prime} r^{\prime}$. The only sourced mode is now $\widetilde{G}_{r^{\prime} r^{\prime}}^{(3) l}$, which is therefore of order $L^{0}$. However, recalling that at a turning point we have $\left(u^{r^{\prime}}\right)^{2} \propto O\left(L^{-2}\right)$, we find that the contribution from this mode to the integral in Eq. (43) is only at order $L^{-2}$, and that the contribution from $\widetilde{G}_{r^{\prime} r^{\prime}}^{(1) l}$ and $\widetilde{G}_{r^{\prime} r^{\prime}}^{(2) l}$ is at still higher order. Therefore, no relevant contributions to $\delta F_{r}^{(\epsilon) l}$ arise for $\alpha^{\prime} \beta^{\prime}=r^{\prime} r^{\prime}$.

In conclusion, when restricting our analysis to the case of a turning point, the problem of calculating the self-force via our mode-sum scheme simplifies considerably: Instead of the three sets of seven equations each for the 21 components required for an arbitrary point of a radial geodesic, one now has to deal with only the three equations (46) and (47) (of which two are coupled and one is closed) for the three components $\widetilde{G}_{t^{\prime} t^{\prime}}^{(1) l}, \widetilde{G}_{t^{\prime} t^{\prime}}^{(2) l}$, and $\widetilde{G}_{t^{\prime} r^{\prime}}^{(2) l}$.

Equations (46) and (47) are solvable in a perturbative manner, using the technique described in detail in Sec. V of Ref. [16]. To apply this technique, one first writes

$$
\widetilde{G}_{\alpha^{\prime} \beta^{\prime}}^{(i) l}=\hat{G}_{\alpha^{\prime} \beta^{\prime}}^{(i) l} \Theta\left(u-u^{\prime}\right) \Theta\left(v-v^{\prime}\right) \quad(i=1,2,3),
$$

where $\Theta$ is the standard step function, acting to confine the support of the Green's function to within the future light cone of the source point $x^{\prime}$. Substituting this expression into Eqs. (46) and (47), one then finds that the new quantities $\hat{G}_{\alpha^{\prime} \beta^{\prime}}^{(i) l}$ (treated as functions of $v, u$ with a fixed source point $\left.v^{\prime}, u^{\prime}\right)$ must satisfy the homogeneous part of these equations anywhere at $v>v^{\prime}$ and $u>u^{\prime}$. One also finds that the value of $\hat{G}_{\alpha^{\prime} \beta^{\prime}}^{(i) l}$ along the null rays $v=v^{\prime}$ and $u=u^{\prime}$ is uniquely determined from Eqs. (46) and (47). [Obtaining these "initial values" for the quantities $\hat{G}_{\alpha^{\prime} \beta^{\prime}}^{(i) l}$ involves the solution of a set of ordinary differential equations along $v=v^{\prime}$ and $u=u^{\prime}$ [29]. In the scalar case, the Green's function was found, in this way, to admit a constant value (of unity) along these initial rays [16]. In the gravitational case, the "initial data" are a bit more complicated, and will be given elsewhere [29].] Thus, in effect, the above procedure converts the original set of Green's function equations into a characteristic initial-data problem for the quantities $\hat{G}_{\alpha^{\prime} \beta^{\prime}}^{(i) l}$, with a unique solution. This unique solution reads, to the relevant order, 


$$
\begin{aligned}
\hat{G}_{t^{\prime} t^{\prime}}^{(1) l}= & J_{0}(z)+\left[f_{2}\left(\Delta_{r}-\Delta_{r^{\prime}}\right) J_{0}(z)-f_{1}\left(\Delta_{r}\right.\right. \\
& \left.\left.+\Delta_{r^{\prime}}\right) z J_{1}(z)\right] L^{-1}+\left\{\left[( \Delta _ { r } - \Delta _ { r ^ { \prime } } ) \left[\left(f_{2}^{2}+f_{4}\right) \Delta_{r}\right.\right.\right. \\
& \left.\left.+f_{4} \Delta_{r^{\prime}}\right]+f_{2}^{2} \Delta_{t}^{2} / 4\right] J_{0}(z)+\left[f_{1}^{2}\left(\Delta_{r}+\Delta_{r^{\prime}}\right)^{2}\right. \\
& \left.-f_{3}\right] z^{2} J_{2}(z) / 2+f_{1}^{2} z^{3} J_{3}(z) / 6+\left[-f_{1} f_{2}\left(\Delta_{r}^{2}-\Delta_{r^{\prime}}^{2}\right)\right. \\
& \left.\left.-f_{3}\left(\Delta_{r^{\prime}}^{2}+\Delta_{r} \Delta_{r^{\prime}}+\Delta_{r^{\prime}}^{2}\right)+f_{5}\right] z J_{1}(z)\right\} L^{-2}+O\left(L^{-3}\right),
\end{aligned}
$$

$\hat{G}_{t^{\prime} t^{\prime}}^{(2) l}=-f^{-1} f_{2}\left(\Delta_{t}+\Delta_{t^{\prime}}\right) J_{0}(z) L^{-1}+\left[f_{6} z J_{1}(z)\right.$

$$
\left.+f_{7} J_{0}(z)\right] \Delta_{r^{\prime}} \Delta_{t} L^{-2}+O\left(L^{-3}\right),
$$

$\hat{G}_{t^{\prime} r^{\prime}}^{(2) l}=J_{0}(z)-f_{1} z J_{1}(z)\left(\Delta_{r}+\Delta_{r^{\prime}}\right) L^{-1}+O\left(L^{-2}\right)$,

where $J_{n}(z)$ are the Bessel functions of the first kind, of order $n$, and the $f_{n}$ 's are radial factors given by $f_{1}$ $=\frac{1}{4} f^{-1 / 2}\left(r f^{\prime}-2 f\right), f_{2}=r f^{\prime} f^{-1 / 2}, f_{3}=r^{2}\left[f^{-1}\left(f^{\prime}\right)^{2}+f^{\prime \prime}\right] / 12$ $+\left(f-r f^{\prime}\right) / 2, \quad f_{4}=r^{2} f^{\prime \prime} / 2, \quad f_{5}=f_{4}+f_{2}^{2} / 2+r f^{\prime} / 2+1 / 8, \quad f_{6}$ $=f^{-1} f_{2}\left(2 f^{1 / 2}-f_{2}\right) / 8$, and $f_{7}=r^{2}\left[f^{-1} f^{\prime \prime}-2 f^{-2}\left(f^{\prime}\right)^{2}\right] / 4$ (all evaluated at $r=r_{0}$ ).

The analysis now proceeds as follows: (i) We substitute solutions (49) in Eq. (42) to obtain the relevant $l$ modes $G_{\alpha \beta \alpha^{\prime} \beta^{\prime}}^{l}$, and then substitute these $l$ modes into Eq. (43). (ii) To be able to carry out the integration in Eq. (43), we next expand all $x^{\prime}$-dependent quantities now appearing in the integrand in powers of $\tau$ about the evaluation point $\tau\left(r_{0}\right)=0$. (This procedure is described in detail, as applied to the analogous scalar case, in Sec. VI of Ref. [16].) (iii) We introduce the "neutral" proper time variable ${ }^{14}$

$$
\bar{\tau}=-\left(L / r_{0}\right) \tau
$$

and write the integrand as an expansion in powers of $1 / L$, with $\bar{\tau}$ held fixed.

Following these manipulations, Eq. (43) takes the form

$$
\begin{aligned}
\delta F_{r}^{(\epsilon) l \pm}= & m^{2} \int_{0}^{L \epsilon / r_{0}}\left[L H_{r}^{(0) \pm}(\bar{\tau})+H_{r}^{(1)}(\bar{\tau})+H_{r}^{(2)}(\bar{\tau}) / L\right. \\
& \left.+O\left(L^{-2}\right)\right] d \bar{\tau}
\end{aligned}
$$

where $H_{r}^{(i)}$ are certain functions of only $\bar{\tau}$ and $r_{0}$ (but do not depend on $l$ otherwise). These functions all have the form of a sum over a few terms $\propto \bar{\tau}^{k} J_{n}(\bar{\tau})$, where $k, n \in \mathbb{N}[$ see, in the analogous scalar case, Eqs. (88)-(93) of Ref. [16]]. The function $H_{r}^{(0)}$ has two different values, denoted in Eq. (51) by $H_{r}^{(0)+}$ and $H_{r}^{(0)-}$, according to whether the derivatives involved in constructing $H_{r}^{(0)}$ are taken from $r \rightarrow r_{0}^{+}$or $r$ $\rightarrow r_{0}^{-}$, respectively. This kind of discontinuity, which shows up only at the leading order in the $1 / L$ expansion, results

\footnotetext{
${ }^{14}$ Note the different notation used in Ref. [16], where the "neutral" proper time variable $\bar{\tau}$ has been denoted by $\lambda$.
}

from differentiating the $\Theta\left(u-u^{\prime}\right) \Theta\left(v-v^{\prime}\right)$ factor appearing in Eq. (48): the contribution coming from the light cone [through the $\delta\left(u-u^{\prime}\right)$ or $\delta\left(v-v^{\prime}\right)$ factors] depends, in its overall sign, upon the direction through which this derivative is taken. This effect is further discussed and illustrated in Sec. IV C of Ref. [16] (see especially Fig. 1 therein).

In terms of the functions $H_{r}^{(i)}$, the regularization parameters are constructed by [16]

$$
\begin{gathered}
A_{r}^{ \pm}=m^{2} \int_{0}^{\infty} H_{r}^{(0) \pm}(\bar{\tau}) d \bar{\tau}, \quad B_{r}=m^{2} \int_{0}^{\infty} H_{r}^{(1)}(\bar{\tau}) d \bar{\tau} \\
C_{r}=m^{2} \int_{0}^{\infty} H_{r}^{(2)}(\bar{\tau}) d \bar{\tau}
\end{gathered}
$$

and

$$
D_{r}=-m^{2} \lim _{\epsilon \rightarrow 0} \sum_{l=0}^{\infty} \int_{L \epsilon / r_{0}}^{\infty}\left[L H_{r}^{(0) \pm}+H_{r}^{(1)}+H_{r}^{(2)} / L\right] d \bar{\tau} .
$$

[Both functions $H_{r}^{(0)+}$ and $H_{r}^{(0)-}$ can be shown [16] to yield the same contribution to the integral in Eq. (53), which is why no \pm sign has been assigned to the parameter $\left.D_{r}.\right]$ The evaluation of the integrals over $\bar{\tau}$ [and of the sum over $l$ in Eq. (53)] is done in a manner completely analogous to the scalar case, as described in Sec. VII of Ref. [16]. Here we merely give the results of this calculation: The parameters $A_{r}^{ \pm}, B_{r}, C_{r}$, and $D_{r}$ are found in our case-a mass particle at a turning point of a radial geodesic_- to be given by

$$
A_{r}^{ \pm}=\mp \frac{m^{2}}{r_{0}^{2}}\left(1-\frac{2 M}{r_{0}}\right)^{-1 / 2}, \quad B_{r}=-\frac{m^{2}}{2 r_{0}^{2}}, \quad C_{r}=D_{r}=0 \text {. }
$$

We comment that the vanishing of the parameter $C_{r}$ is necessary to assure consistency of our entire scheme: otherwise, the parameter $D_{r}$ would have been indefinite (this point is explained in Sec. VII D of Ref. [16]). ${ }^{15}$

The values derived here for the regularization parameters find support from a recent numerical analysis carried out by Lousto [12], who numerically calculated the bare modes of the force, $F_{\alpha}^{l(\text { bare })}$, for a radially moving particle in Schwarzschild spacetime (as part of the implementation of a different regularization scheme), and found that these modes indeed show a large- $l$ behavior of the form indicated in Eq. (34) above. Furthermore, the analytical expressions derived here for the coefficients $A_{r}^{ \pm}, B_{r}$, and $C_{r}$ show a perfect agreement with the numerically derived coefficients [29]. In addition, our result of a vanishing parameter $D_{\alpha}$ confirms, in the cases studied here, Lousto's result based on his proposed zeta-function regularization. (Although Lousto's numerical

\footnotetext{
${ }^{15}$ Interestingly, the parameter values [Eq. (54)] coincide with the values obtained for the scalar self-force acting on a particle of scalar charge $q=m$, at a turning point of a radial geodesic in Schwarzschild spacetime-see Ref. [16].
} 
calculations were carried out in a different gauge-in the RW gauge rather than in the harmonic gauge-this should not alter the values of the regularization parameters, as we explain in Ref. [10].) Finally, we mention that the expressions derived here for $A_{r}^{ \pm}$and $B_{r}$ also agree with the analytic values obtained recently using a different formalism, in which the self-force was derived from the so-called Moncrief wave form $\psi[29]{ }^{16}$

In conclusion of this section, we have found that the $r$ component of the regularized self-force at a turning point of a radial geodesic in Schwarzschild spacetime is given by above Eq. (38), where the parameter $B_{r}$ is given in Eq. (54), and where $\bar{F}_{r}^{l \text { (bare) }}$ are the (sided average) $l$ modes of the bare force. These bare modes are derived [through the second equality of Eq. (28)] from the metric perturbation in the harmonic gauge. For practical use, it would be desirable to express our result in a more useful gauge (e.g., in the RW gauge). This shall be done in a forthcoming paper [10], as part of a more general discussion of the gauge issue in the context of the gravitational self-force problem.

\section{SUMMARY AND DISCUSSION}

In this paper we have generalized the scheme of modesum regularization, previously applied only in the scalar case, to the problem of calculating the gravitational selfforce on a mass particle. The proposed scheme offers a practical way for implementing the formal prescription developed by MST and QW, even in strong-field calculations.

Within the mode-sum scheme, the basic formula for constructing the (harmonic-gauge related) gravitational selfforce is given by

$$
F_{\alpha}^{H}=\sum_{l=0}^{\infty}\left(\left[F_{\alpha}^{l(\text { bare })}\right]^{H}-A_{\alpha} L-B_{\alpha}-C_{\alpha} / L\right)-D_{\alpha}
$$

where $L \equiv l+1 / 2$ and the label $H$ indicates a quantity associated with the metric perturbation in the harmonic gauge. To apply this formula in actual calculations (i.e., for a certain orbit on a specific background), one needs to be provided with (i) the bare force modes $\left[F_{\alpha}^{l \text { (bare) }}\right]^{H}$ and (ii) the values of the four regularization parameters $A_{\alpha}, B_{\alpha}, C_{\alpha}$, and $D_{\alpha}$. To obtain the bare modes $\left[F_{\alpha}^{l \text { (bare })}\right]^{H}$, one first calculates the multipole modes of the metric perturbation in the harmonic gauge, and then uses the relation

$$
\left[F_{\alpha}^{l(\text { bare })}\right]^{H}=m k_{\alpha}^{\beta \gamma \delta}\left[\bar{h}_{\beta \gamma}^{l}\right]_{; \delta}^{H},
$$

where $k^{\alpha \beta \gamma \delta}$ is the tensor given in Eq. (5), and $\left[\bar{h}_{\beta \gamma}^{l}\right]^{H}$ is the $l$ mode of the trace-reversed metric perturbation (in the harmonic gauge). Whereas this part of the analysis-the deriva-

\footnotetext{
${ }^{16}$ So far, agreement has been achieved for both one-sided values of $A_{r}$ and $B_{r}$, as well as for the average value of $C_{r}$. Currently, we obtain, using Moncrief's formalism, that although the averaged $C_{r}$ vanishes (as in our present analysis), the one-sided values of $C_{r}$ fail to vanish. It is most likely that this preliminary result is erroneous. This point awaits further examination.
}

tion of the bare modes-typically involves a numerical analysis, the derivation of the regularization parameters may be carried out analytically, by means of local analysis of the Green's function. This kind of local analysis was described and demonstrated in Sec. V, where we constructed all necessary regularization parameters for two simple cases.

The first case examined in Sec. V was the trivial test case of a static particle in flat spacetime. Here the mode-sum scheme easily reproduced the obvious result of a vanishing self-force. We then calculated the regularization parameters for the case of a mass particle at a turning point of a radial geodesic in Schwarzschild spacetime. The values of these parameters were given in Eq. (54). These values find support from a recent numerical analysis by Lousto $[12,29]$.

Our calculation scheme- like the formal prescription by MST and QW on which it relies-is formulated within the harmonic gauge. In an accompanying paper [10] we explore the gauge dependence of the gravitational self-force, and reformulate our scheme in terms of other gauges-ones more commonly adopted in perturbation analysis (such as the RW gauge). We then conclude that an expression of the form of Eq. (55) is applicable for calculating the self-force in any specific gauge $G$ (as long as the the self-force attains a definite finite value in that gauge), by just replacing the harmonic gauge modes $\left[F_{\alpha}^{l \text { (bare) }}\right]^{H}$ on the right-hand side of Eq. (55) with the $G$ gauge modes $\left[F_{\alpha}^{l(\text { bare })}\right]^{G}$ - the ones derived from the $G$ gauge metric perturbation using Eq. (56), with $H \rightarrow G$. The analysis of Ref. [10] further tells us that the regularization parameters in Eqs. (55) should not carry any gauge label: these parameters are "gauge invariant," in the sense that they are always to be derived from the Green's function associated with the harmonic-gauge wave operator [the one defined through Eq. (6)], irrespective of the gauge in which the bare modes are calculated. In particular, we find that the values specified in Eq. (54) are valid under any gauge.

\section{Further applications of the proposed calculation scheme}

The analysis of Sec. III provides formal tools for calculating the gravitational self-force in any orbit on a Schwarzschild background. For any such orbit, the regularization parameters may be derived by local analysis of the Green's function modes, based on the separated system of equations (24) [supplemented by Eqs. (26)] —as demonstrated in Sec. V. It should be noted, however, that in more general cases than the simple ones considered here, the derivation of the regularization parameters shall require much more technical effort: Here we only had to deal with one equation for one Green's function component (in the case of a static particle in flat spacetime), or with three coupled equations for three components (in the case of a particle at a turning point of a radial geodesic). We shall have to face three sets of 7 coupled equations each for a total of 21 components already for an arbitrary point of a radial geodesic. In general cases, one would have to deal with up to 58 equations (seven sets of seven coupled equations each for even perturbation modes, and three sets of three equations each for odd perturbation modes). 
To fully implement the regularization scheme and derive the self-force, one also needs to calculate the bare modes of the force. This was already done by Lousto [12], using Moncrief's formalism [30], for radial geodesic motion in Schwarzschild spacetime. In his analysis, Lousto numerically calculated the (one-sided averaged) values of $A_{\alpha}, B_{\alpha}$, and $C_{\alpha}$, and presumed a null value for $D_{\alpha}$ based on a proposed zeta-function regularization scheme. To provide a full theoretical basis for Lousto's results in the radial motion case (and verify its consistency with MSTQW regularization) we intend to calculate analytically, using our mode-sum scheme, all four parameters $A_{\alpha}, B_{\alpha}, C_{\alpha}$, and $D_{\alpha}$ for an arbitrary point of a radial geodesic [29]. Then, the next natural step would be to consider more general, nonradial orbits. This would require a considerable amount of both analytical work (deriving the regularization parameters) and numerical work (calculating the bare modes of the force by solving the metric perturbation equations).

We finally comment on the applicability of our mode-sum regularization scheme to orbits in Kerr spacetime. Although the theoretical basis for applying our scheme for nonspherically symmetric backgrounds has not been developed yet (even in the scalar case), we believe that a properly generalized version of this scheme could, eventually, cope with the Kerr case as well. Such a generalization could still be based on MST and QW's formal prescription [see Eq. (4)], which applies for any vacuum spacetime. The main obstacle in redesigning our scheme for the Kerr case would be, of course, the nonseparability of the metric perturbation and Green's function into multipole modes in the time domain (such a separation has been a necessary step when executing our scheme in spherically symmetric cases). This difficulty would make both the analytical and numerical parts of the mode-sum scheme more challenging: The bare modes of the force would usually have to be calculated in the frequency domain (using the Teukolsky-Chrzanowski formalism [28]), and then appropriately summed over Fourier frequencies. As to the analytical part of the scheme, it seems to us that, with the use of an appropriate local perturbation analysis, enough information for constructing the regularization parameters could be extracted from the time-domain Green's function equations, even without fully separating these equations.

\section{ACKNOWLEDGMENTS}

I am very grateful to Amos Ori for his guidance throughout the execution of this research project, and for many interesting discussions. It is a pleasure to thank Eric Poisson for his valuable comments on an early version of this manuscript. This research was partially supported by the United States-Israel Binational Science Foundation; and by the European Community program IHP-MCIF-99-1 under Contract No. HPMF-CT-2000-00851.

\section{APPENDIX: COUPLING OF GREEN'S FUNCTION'S COMPONENTS (SCHWARZSCHILD SPACETIME)}

In this appendix we explicitly give the coupling terms appearing in the Green's function equation (8), for the
Schwarzschild spacetime case (and using Schwarzschild coordinates). In the following expressions $f \equiv 1-2 M / r$ and a prime denotes $d / d r$ :

$$
\begin{aligned}
\mathcal{A}_{t t}^{\mu \nu} \bar{G}_{\mu \nu}= & {\left[f^{-1}\left(f^{\prime}\right)^{2} / 2-2 f^{\prime} \partial_{r}\right] \bar{G}_{t t}+2 f^{\prime} \partial_{t} \bar{G}_{t r}+\left[f^{2} f^{\prime \prime}\right.} \\
& \left.-f\left(f^{\prime}\right)^{2} / 2\right] \bar{G}_{r r}+r^{-3} f f^{\prime}\left(\bar{G}_{\theta \theta}+\sin ^{-2} \theta \bar{G}_{\varphi \varphi}\right),
\end{aligned}
$$

$$
\begin{aligned}
\mathcal{A}_{t r}^{\mu \nu} \bar{G}_{\mu \nu}= & {\left[f^{\prime \prime}-f^{-1}\left(f^{\prime}\right)^{2}-2 r^{-2} f\right] \bar{G}_{t r}+f^{-2} f^{\prime} \partial_{t} \bar{G}_{t t} } \\
& +f^{\prime} \partial_{t} \bar{G}_{r r}-2 r^{-3}\left(\partial_{\theta}+\cot \theta\right) \bar{G}_{t \theta} \\
& -2 r^{-3} \sin ^{-2} \theta \partial_{\varphi} \bar{G}_{t \varphi}
\end{aligned}
$$

$$
\begin{aligned}
\mathcal{A}_{r r}^{\mu \nu} \bar{G}_{\mu \nu}= & {\left[2 f^{\prime} \partial_{r}-4 f / r^{2}+f^{-1}\left(f^{\prime}\right)^{2} / 2\right] \bar{G}_{r r}+2 f^{-2} f^{\prime} \partial_{t} \bar{G}_{t r} } \\
& +\left[f^{-2} f^{\prime \prime}-f^{-3}\left(f^{\prime}\right)^{2} / 2\right] \bar{G}_{t t}-4 r^{-3}\left(\partial_{\theta}+\cot \theta\right) \bar{G}_{r \theta} \\
& -4 r^{-3} \sin ^{-2} \theta \partial_{\varphi} \bar{G}_{r \varphi}+\left(2 r^{-4}-r^{-3} f^{-1} f^{\prime}\right)\left(\bar{G}_{\theta \theta}\right. \\
& \left.+\sin ^{-2} \theta \bar{G}_{\varphi \varphi}\right),
\end{aligned}
$$

$$
\begin{aligned}
\mathcal{A}_{t \theta}^{\mu \nu} \bar{G}_{\mu \nu}= & {\left[f^{\prime} / r-\left(f^{\prime}+2 f / r\right) \partial_{r}-r^{-2} \sin ^{-2} \theta\right] \bar{G}_{t \theta}+f^{\prime} \partial_{t} \bar{G}_{r \theta} } \\
& +2(f / r) \partial_{\theta} \bar{G}_{t r}-2 r^{-2} \sin ^{-2} \theta \cot \theta \partial_{\varphi} \bar{G}_{t \varphi}, \quad \text { (A4) }
\end{aligned}
$$

$$
\mathcal{A}_{r \theta}^{\mu \nu} \bar{G}_{\mu \nu}=\left[\left(f^{\prime}-2 f / r\right) \partial_{r}-4 f / r^{2}-r^{-2} \sin ^{-2} \theta\right] \bar{G}_{r \theta}
$$

$$
\begin{aligned}
& +2(f / r) \partial_{\theta} \bar{G}_{r r}+f^{-2} f^{\prime} \partial_{t} \bar{G}_{t \theta}-2 r^{-3}\left(\partial_{\theta}\right. \\
& +\cot \theta) \bar{G}_{\theta \theta}+2 r^{-3} \sin ^{-2} \theta \cot \theta \bar{G}_{\varphi \varphi} \\
& -2 r^{-2} \sin ^{-2} \theta \cot \theta \partial_{\varphi} \bar{G}_{r \varphi}-2 r^{-3} \sin ^{-2} \theta \partial_{\varphi} \bar{G}_{\theta \varphi},
\end{aligned}
$$

$$
\begin{aligned}
\mathcal{A}_{\theta \theta}^{\mu \nu} \bar{G}_{\mu \nu}= & {\left[-4(f / r) \partial_{r}+2\left(f / r^{2}\right)-2 r^{-2} \sin ^{-2} \theta\right] \bar{G}_{\theta \theta} } \\
& +r f^{-1} f^{\prime} \bar{G}_{t t}+\left(2 f^{2}-r f f^{\prime}\right) \bar{G}_{r r}+4(f / r) \partial_{\theta} \bar{G}_{r \theta} \\
& +2\left(r^{-2} \cot ^{2} \theta+f^{\prime} / r\right) \sin ^{-2} \theta \bar{G}_{\varphi \varphi} \\
& -4 r^{-2} \sin ^{-2} \theta \cot \theta \partial_{\varphi} \bar{G}_{\theta \varphi}
\end{aligned}
$$

$$
\begin{aligned}
\mathcal{A}_{\varphi \varphi}^{\mu \nu} \bar{G}_{\mu \nu}= & {\left[-4(f / r) \partial_{r}-4 r^{-2} \cot \theta \partial_{\theta}+2\left(f / r^{2}\right)\right.} \\
& \left.+2 r^{-2} \cot ^{2} \theta\right] \bar{G}_{\varphi \varphi}+r f^{-1} f^{\prime} \sin ^{2} \theta \bar{G}_{t t}+\left(2 f^{2}\right. \\
& \left.-r f f^{\prime}\right) \sin ^{2} \theta \bar{G}_{r r}+4(f / r) \sin \theta \cos \theta \bar{G}_{r \theta} \\
& +4(f / r) \partial_{\varphi} \bar{G}_{r \varphi}+\left[2 r^{-2} \cos ^{2} \theta\right. \\
& \left.+2\left(f^{\prime} / r\right) \sin ^{2} \theta\right] \bar{G}_{\theta \theta}+4 r^{-2} \cot \theta \partial_{\varphi} \bar{G}_{\theta \varphi},
\end{aligned}
$$

$$
\begin{aligned}
\mathcal{A}_{t \varphi}^{\mu \nu} \bar{G}_{\mu \nu}= & {\left[-\left(f^{\prime}+2 f / r\right) \partial_{r}-2 r^{-2} \cot \theta \partial_{\theta}+2 f^{\prime} / r\right] \bar{G}_{t \varphi} } \\
& +2 r^{-2} \cot \theta \partial_{\varphi} \bar{G}_{t \theta}+2(f / r) \partial_{\varphi} \bar{G}_{t r}+f^{\prime} \partial_{t} \bar{G}_{r \varphi},
\end{aligned}
$$




$$
\begin{aligned}
\mathcal{A}_{r \varphi}^{\mu \nu} \bar{G}_{\mu \nu}= & {\left[\left(f^{\prime}-2 f / r\right) \partial_{r}-4 f / r^{2}-2 r^{-2} \cot \theta \partial_{\theta}\right] \bar{G}_{r \varphi} } \\
& +2(f / r) \partial_{\varphi} \bar{G}_{r r}+f^{-2} f^{\prime} \partial_{t} \bar{G}_{t \varphi} \\
& -2 r^{-3} \sin ^{-2} \theta \partial_{\varphi} \bar{G}_{\varphi \varphi}+2 r^{-2} \cot \theta \partial_{\varphi} \bar{G}_{r \theta} \\
& -2 r^{-3}\left(\partial_{\theta}+\cot \theta\right) \bar{G}_{\theta \varphi},
\end{aligned}
$$

$$
\begin{aligned}
A_{\theta \varphi}^{\mu \nu} \bar{G}_{\mu \nu}= & {\left[-4(f / r) \partial_{r}-2 r^{-2} \cot \theta \partial_{\theta}-3 r^{-2} \cot ^{2} \theta-2 f^{\prime} / r\right.} \\
& \left.+(2 f-1) / r^{2}\right] \bar{G}_{\theta \varphi}+2(f / r) \partial_{\varphi} \bar{G}_{r \theta} \\
& +2(f / r)\left(\partial_{\theta}-2 \cot \theta\right) \bar{G}_{r \varphi}+2 r^{-2} \cot \theta \partial_{\varphi} \bar{G}_{\theta \theta} \\
& -2 r^{-2} \sin ^{-2} \theta \cot \theta \partial_{\varphi} \bar{G}_{\varphi \varphi} .
\end{aligned}
$$

[1] P.A.M. Dirac, Proc. R. Soc. London, Ser. A 167, 148 (1938).

[2] See, e.g., E.E. Flanagan and R.M. Wald, Phys. Rev. D 54, 6233 (1996); for a pedagogical introduction to the ALD equation see E. Poisson, gr-qc/9912045.

[3] B.S. DeWitt and R.W. Brehme, Ann. Phys. (N.Y.) 9, 220 (1960).

[4] LISA webpage: http://lisa.jpl.nasa.gov/

[5] See, e.g., S.W. Leonard and E. Poisson, Phys. Rev. D 56, 4789 (1997), and references therein.

[6] Y. Mino, M. Sasaki, and T. Tanaka, Phys. Rev. D 55, 3457 (1997).

[7] T.C. Quinn and R.M. Wald, Phys. Rev. D 56, 3381 (1997).

[8] J. Hadamard, Lectures on Cauchy's Problem in Linear Partial Differential Equations (Dover, New York, 1952).

[9] T.C. Quinn, Phys. Rev. D 62, 064029 (2000).

[10] L. Barack and A. Ori, gr-qc/0107056.

[11] M.J. Pfenning and E. Poisson, gr-qc/0012057.

[12] C.O. Lousto, Phys. Rev. Lett. 84, 5251 (2000).

[13] H. Nakano and M. Sasaki, Prog. Theor. Phys. 105, 197 (2001).

[14] S. Detweiler, Phys. Rev. Lett. 86, 1931 (2001).

[15] L. Barack and A. Ori, Phys. Rev. D 61, 061502(R) (2000).

[16] L. Barack, Phys. Rev. D 62, 084027 (2000).

[17] L.M. Burko, Class. Quantum Grav. 17, 227 (2000).
[18] L.M. Burko, Phys. Rev. Lett. 84, 4529 (2000).

[19] L. Barack and L.M. Burko, Phys. Rev. D 62, 084040 (2000).

[20] L.M. Burko, Y.-T. Liu, and Y. Soen, Phys. Rev. D 63, 024015 (2001).

[21] L.M. Burko and Y.-T. Liu, Phys. Rev. D 64, 024006 (2001).

[22] C.W. Misner, K.S. Thorne, and J.A. Wheeler, Gravitation (Freeman, San Francisco, 1970).

[23] Note the mistake in Eqs. (49)-(51) of Ref. [7], where the trace-reversed Green's function has been used instead of the Green's function associated with the metric perturbation itself.

[24] T. Regge and J.A. Wheeler, Phys. Rev. 108, 1063 (1957). Note that the basis of tensor harmonics introduced by Regge and Wheeler is not orthogonal.

[25] F.J. Zerilli, J. Math. Phys. 11, 2203 (1970); Phys. Rev. D 2, 2141 (1970).

[26] F.J. Zerilli, Phys. Rev. D 2, 2141 (1970).

[27] For a comprehensive review of tensor multipole expansions, see K.S. Thorne, Rev. Mod. Phys. 52, 299 (1980).

[28] P.L. Chrzanowski, Phys. Rev. D 11, 2042 (1975).

[29] L. Barack and C. O. Lousto (unpublished). Some preliminary results of this work are given in C.O. Lousto, Proceedings of the 3rd International LISA Symposium, gr-qc/0010007.

[30] V. Moncrief, Ann. Phys. (N.Y.) 88, 323 (1974). 